Check for updates

Cite this: Phys. Chem. Chem. Phys., 2020, 22, 20311

Received 29th July 2020 ,

Accepted 31st August 2020

DOI: $10.1039 / \mathrm{d} 0 \mathrm{cp04033a}$

rsc.li/pccp

\section{The electronic structure of the aqueous permanganate ion: aqueous-phase energetics and molecular bonding studied using liquid jet photoelectron spectroscopy $\dagger$}

Karen D. Mudryk, (D) ${ }^{a b}$ Robert Seidel, (D) ${ }^{c d}$ Bernd Winter (DD ${ }^{e}$ and lain Wilkinson (DD *a

\section{Introduction}

The aqueous-phase permanganate ion, $\mathrm{MnO}_{4}{ }^{-}$(aq.), is well-known for its properties as a strong oxidizing agent over a broad range of $\mathrm{pH}$, acting as a redox precursor of several manganese species with different oxidation states, namely the $\mathrm{MnO}_{4}{ }^{2-}$ (aq.), $\mathrm{MnO}_{4}{ }^{3-}{ }_{\text {(aq.) }}, \quad \mathrm{MnO}_{2(\mathrm{~s})}, \quad \mathrm{Mn}\left(\mathrm{H}_{2} \mathrm{O}\right)_{6}{ }^{3+}{ }_{\text {(aq.) }}, \quad$ and $\mathrm{Mn}\left(\mathrm{H}_{2} \mathrm{O}\right)_{6}{ }^{2+}$ (aq.) complexes. ${ }^{1}$ Thus, $\mathrm{MnO}_{4}{ }^{-}$(aq.) is considered a versatile oxidizer

\footnotetext{
${ }^{a}$ Locally-Sensitive \& Time-Resolved Spectroscopy, Helmholtz-Zentrum Berlin für Materialien und Energie, Hahn-Meitner-Platz 1, D-14109 Berlin, Germany. E-mail: iain.wilkinson@helmholtz-berlin.de

${ }^{b}$ Fachbereich Physik, Freie Universität Berlin, Arnimallee 14, D-14195 Berlin, Germany

${ }^{c}$ Operando Interfacial Photochemistry, Helmholtz-Zentrum Berlin für Materialien und Energie, Albert-Einstein-Strasse 15, D-12489 Berlin, Germany

${ }^{d}$ Fachbereich Chemie, Humboldt-Universität zu Berlin, Brook-Taylor-Strasse 2, D-12489 Berlin, Germany

${ }^{e}$ Fritz-Haber-Institut der Max-Planck-Gesellschaft, Faradayweg 4-6, D-14195 Berlin, Germany

$\dagger$ Electronic supplementary information (ESI) available. See DOI: 10.1039/d0cp04033a
}

suitable for green chemistry, ${ }^{2}$ oxidation of micro-pollutants, ${ }^{3}$ detection of organic compounds, ${ }^{4}$ water disinfection, ${ }^{5-7}$ and synthesis of energy materials. ${ }^{8}$ This transition-metal complex has also been applied in energy conversion and storage systems, such as $\mathrm{MnO}_{4}{ }^{-}{ }_{\text {(aq.) }} / \mathrm{MnO}_{4}{ }^{2-}{ }_{\text {(aq.) }}$ redox flow batteries ${ }^{9}$ and microbial fuel cells. ${ }^{10}$

The aforementioned applications rely on the same fundamental process, that is, an electron-transfer reaction between a reduced solute or an electrode (acting as the electron donor) and $\mathrm{MnO}_{4}{ }^{-}$(aq.) (acting as the electron acceptor). During this type of reaction, the electronic and nuclear geometric configuration of both the donor and acceptor, as well as the dielectric and microscopic electronic polarization of the surrounding solvent molecules, are generally changed. ${ }^{11}$ The energetics associated with these changes can be expressed as a reorganization energy which can be broken down into vibrational, $\lambda_{\mathrm{i}}$, and solvational, $\lambda_{0}$, components. It has previously been demonstrated that (total) oxidative reorganisation energies, $\lambda_{\text {ox }}$, may be directly extracted from photoelectron spectroscopy (PES) measurements, ${ }^{12-14}$ as reported here. While dielectric properties of the solvent are 
required to calculate $\lambda_{0}$, detailed knowledge of the electronic structure of the reactants in the reaction medium is a prerequisite to calculate $\lambda_{\mathrm{i}} \cdot{ }^{12-16}$ Experimentally, such electronic structure information may be garnered using PES techniques. ${ }^{17-19}$

Solute- and solvent-induced electronic polarization effects affect the reaction energetics and pathways that facilitate a redox process, ${ }^{11}$ with charge distributions within the solute and in the solution more generally determining the kinetics and mechanisms involved in the aforementioned applications. In an aqueous solution, generally, the electronic structure of the solute and the solvent are correlated and influenced by each other. $^{20,21}$ Geometric structural changes may also arise due to solute-solvent interactions. ${ }^{2-24}$ Furthermore, in transitionmetal complexes, the redox potential varies depending on the electronic charge distributions/molecular bonding interactions between the metal centre and its ligands. ${ }^{17,25,26}$ Hence, aqueous-phase electronic structure studies are expected to provide an abundance of insights into the redox behaviour of $\mathrm{MnO}_{4}{ }^{-}$(aq.) and its role in determining the efficiency of associated electron-transfer processes.

Liquid microjet-based soft X-ray PES (XPS) allows valenceand core-level ionization energies of aqueous solutes and the associated solvent to be determined on an absolute energy scale. ${ }^{17,27}$ The technique also allows aqueous-gas interfaces or (predominantly) bulk-liquid environments to be probed through tuning of ionising photon energies. Thereby the probing depth of the (photo)electrons in the solution is varied, enabling preferential surface- versus predominantly bulksensitive probing. ${ }^{27-29}$ Further electronic structure insights may be garnered by performing resonantly-enhanced photoelectron spectroscopy (RPES) experiments. ${ }^{17,30,31}$ Through excitation at core-to-virtual-valence resonances, signals from solutes with relatively low ionization cross-sections or local concentrations can be selectively enhanced and detected. ${ }^{32}$ Furthermore, the energetics of resonant (valence) photoelectron signals generated following core-to-virtual-valence excitation and solute intramolecular relaxation allow the atomic parentages of specific molecular bonding interactions to be interrogated. ${ }^{17}$ Additionally, such experiments potentially access non-local relaxation phenomena, such as Intermolecular Coulombic Decay (ICD), and intermolecular electronic interactions between solute and solvent molecules. ${ }^{33}$ Here we utilize these salient features of aqueous-phase PES to elucidate the electronic structure of the aqueous permanganate ion. We aim to characterize both the solute's complex intra-molecular electronic structure as well as identify signatures of electronic interactions between the permanganate ions and hydrating water molecules.

To our knowledge, all previous PES studies of the permanganate ion have focused on gaseous $\mathrm{MnO}_{4}{ }^{-34}$ and solid $\mathrm{KMnO}_{4}{ }^{35-38}$ samples. By extending such studies to the aqueous phase, we thus provide experimental insight into the effect of hydration on the electronic structure and redox properties of the widely applied aqueous permanganate ion. Specifically, we report soft X-ray liquid microjet non-resonant and resonant PES experiments from $\mathrm{MnO}_{4}^{-}$aqueous solutions. We present absolute binding energies (BEs) of the valence orbitals and of the $\mathrm{O} 1 \mathrm{~s}$ and $\mathrm{Mn} 2 \mathrm{p}_{3 / 2,1 / 2}$ core levels (with respect to the vacuum level). We also explore the effect of interfacial versus liquid-bulk hydration and counter-ion $\left(\mathrm{Na}^{+}\right.$versus $\left.\mathrm{K}^{+}\right)$on the measured valence BEs at relatively low permanganate ion concentrations $(\sim 0.2 \mathrm{M})$. In addition, we use $\mathrm{Mn} \mathrm{L}_{\mathrm{II}, \mathrm{III}}{ }^{-}$and $\mathrm{O}$ K-pre-edge RPES measurements to confirm and expand on direct PES determinations of the $\mathrm{MnO}_{4}{ }^{-}$BEs and elucidate ionisation energies that are otherwise difficult to extract. The RPES results are also used to identify the atomic parentages and confirm hybridizations of a number of the valence molecular orbitals. ${ }^{39-41}$ Furthermore, intermolecular solute and solvent electronic coupling phenomena are identified through detection of resonant ICD processes following soft X-ray photoexcitation. Our cumulative results are used to construct an aqueous-phase molecular orbital diagram for the $\mathrm{MnO}_{4}{ }^{-}$(aq.) anion, as well as an electronic state energy level diagram for the $\mathrm{MnO}_{4}^{-}$(aq.) anion/ $\mathrm{MnO}_{4}{ }^{\bullet}$ (aq.) radical system. We additionally infer (valence ionisation) reorganisation energies of oxidation, $\lambda_{\mathrm{ox}}$, and adiabatic ionisation energies (AIEs, equivalent to the Gibbs free energy of oxidation in the case of the lowest energy ionising transition) of the $\mathrm{MnO}_{4}{ }^{-}$(aq.) ion. Furthermore, we estimate the vertical electron affinity (VEA) of the $\mathrm{MnO}_{4}{ }^{\bullet}$ (aq.) radical, and the Gibbs free energy of hydration, $\Delta G_{\text {hyd }}$, of isolated $\mathrm{MnO}_{4}{ }^{-}$. Combinatorially, our direct PES and RPES results allow us to produce a comprehensive compilation of the electronic energy level structure of the permanganate ion in bulk aqueous solution and at the aqueous-solution-gas interface.

\section{Experimental}

\section{Measurements}

Experiments were primarily performed at the U49/2 PGM-1 beamline ${ }^{42}$ with the SOL ${ }^{3}$ PES end-station ${ }^{43}$ at the BESSY II synchrotron facility (Helmholtz-Zentrum Berlin für Materialien und Energie). Select experiments were repeated at the P04 beamline $^{44}$ with the EASI end-station ${ }^{45}$ at the PETRA III synchrotron facility (Deutsches Elektronen-Synchrotron). $\mathrm{MnO}_{4}{ }^{-}$(aq.) solutions were prepared at room temperature, either by diluting aliquots of a commercial $\mathrm{NaMnO}_{4(\text { aq.) }}$ solution (Aldrich, $40 \mathrm{wt} \%$ ) or by dissolving $\mathrm{KMnO}_{4}$ crystals (Roth, $\geq 99 \%$ and Fluka, $\geq 99 \%)$ in millipure water $(18.2 \mathrm{M} \Omega \mathrm{cm}$ resistivity at $25{ }^{\circ} \mathrm{C}$ ). Following preparation, all sample reservoirs containing $\mathrm{MnO}_{4}{ }^{-}$(aq.) solutions were shielded from ambient light to prevent photochemical reactions. ${ }^{46}$ After ultrasonic degassing, the samples were introduced into the liquid-jet-synchrotron-beam interaction vacuum chamber of the end-stations as horizontal, free-flowing liquid microjets. ${ }^{30}$ The solutions were injected at

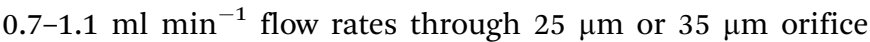
diameter quartz capillary nozzles using high-performance liquid chromatography (HPLC) pumps. All sample delivery materials were chemically inert towards $\mathrm{MnO}_{4}{ }^{-}$(aq.) . The jet was electrically grounded to the interaction chamber by means of a gold wire immersed in the solution close to the injection point. A cooling system connected to the liquid jet nozzle 
holder allowed the temperature of the solution to be regulated prior to injection. Generally, both the temperature and sample flow rate were optimized to maximize jet stability during measurements. However, specific sample temperatures (in the $280 \pm 3 \mathrm{~K}$ range) were adopted in situations where PES peak spectral widths are compared or used to derive additional parameters. The pressures in the interaction chambers were maintained between $5 \times 10^{-4}$ and $1 \times 10^{-3}$ mbar using a combination of turbo molecular pumping $\left(\sim 2000\right.$ or $\sim 2700 \mathrm{~L} \mathrm{~s}^{-1}$ pumping speed for water vapour in the SOL ${ }^{3} \mathrm{PES}$ and EASI instruments, respectively) and two (SOL $\left.{ }^{3} \mathrm{PES}\right)$ or three (EASI) liquid nitrogen-filled cold traps $\left(\sim 4.5 \times 10^{4} \mathrm{~L} \mathrm{~s}^{-1}\right.$ pumping speed for water vapour per trap in both instruments). ${ }^{43,45}$

The U49/2 PGM-1 beamline provided linearly, horizontallypolarized light and a focal spot size of approximately $100 \times$ $80 \mu \mathrm{m}^{2}$ (horizontal $\times$ vertical). ${ }^{42}$ The $\mathrm{P} 04$ beamline provided circularly-polarized light and a reduced vertical focal spot size

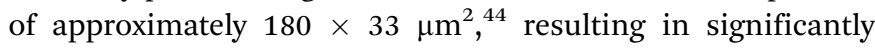
diminished gas-phase contributions to the recorded spectra. The beamlines were protected from the liquid samples introduced into the end-station interaction chambers using windowless differential pumping chamber assemblies. ${ }^{43,45}$ The photon flux at U49/2 PGM-1 ${ }^{42}$ (measured after the last focusing mirror in the beamline) is of the order of $10^{13} \mathrm{ph} \mathrm{s}^{-1}$ in the $85-500 \mathrm{eV}$ photon energy range or $10^{12} \mathrm{ph} \mathrm{s}^{-1}$ in the $500-1500 \mathrm{eV}$ photon energy range, with a slightly higher photon flux available in the latter energy range at the sample position at P04. ${ }^{44}$ All spectra were recorded at maximum photon flux, with corresponding peak intensities of $\sim 10^{3} \mathrm{~W} \mathrm{~cm}^{-2}$ under our experimental conditions. The associated absorbed X-ray doses (D) on the samples were of the order of $10^{-3}$ Gy. As expected, spectra recorded at $635 \mathrm{eV}$ photon energy and full and reduced $(50 \%$ and $10 \%)$ flux showed no indication of sample radiation damage.

The photoelectrons emitted from the liquid jet and the surrounding gas-phase molecules were detected using differentiallypumped hemispherical electron analysers (Scienta Omicron R4000 HiPP-2 in the SOL ${ }^{3}$ PES setup, ${ }^{43}$ or HiPP-3 in the EASI instrument ${ }^{45}$ ). The electron collection axis of the analyser was set orthogonal or at magic angle to the light propagation and light polarization axes in all experiments. The liquid jet propagated orthogonally to both the light propagation and analyser electron collection axes. The analyser parameters were set to maximize electron collection by adopting an analyser entrance slit aperture larger than the limiting aperture located at the exit of the analyser pre-lens and the entrance of the hemisphere. ${ }^{43,47}$ The liquid jet position was optimized by maximizing the count rate of the lowest energy ionising transition $\left(1 b_{1}{ }^{-1}\right.$ peak) of liquid water (in experiments performed at photon energies lower than $540 \mathrm{eV}$ ) or of the liquid water Auger electron signal (in experiments performed at photon energies higher than $540 \mathrm{eV}$ ), thereby minimizing the gas-phase contributions to the spectra. The energy resolution of the U49/2 PGM-1 beamline and SOL ${ }^{3}$ PES end-station experimental setup was determined by recording gas-phase Ar XPS spectra under identical beamline and analyser conditions to those adopted in the liquid jet experiments. Voigt profile fits to Ar $3 p$ or $2 p$ XPS spectra allowed us to determine instrumental resolutions of $\sim 53 \mathrm{meV}, \sim 310 \mathrm{meV}$, and $\sim 400 \mathrm{meV}$ at $150 \mathrm{eV}, 530 \mathrm{eV}$, and $638 \mathrm{eV}$, respectively. In the P04 beamline and EASI endstation experiments, total energy resolutions of $\sim 410 \mathrm{meV}, \sim 460 \mathrm{meV}$, and $\sim 480 \mathrm{meV}$ were estimated at $635 \mathrm{eV}, 1135 \mathrm{eV}$, and $1251 \mathrm{eV}$ photon energies, respectively.

Non-resonant, surface-sensitive valence photoelectron spectra were recorded at photon energies of $150 \mathrm{eV}$. This corresponds to a $\sim 130 \mathrm{eV}$ photoelectron kinetic energy (KE) and probing of all signal components at similar depths of $\sim 1 \mathrm{~nm}^{28,29,48}$ Surface-and-bulk-sensitive valence photoelectron measurements were performed at a $635 \mathrm{eV}$ photon energy resulting in $\sim 600 \mathrm{eV}$ KEs. Corresponding electron effective attenuation lengths (EALs) are estimated to be $\sim 4 \mathrm{~nm}$, with the associated signals predominantly attributed to the aqueous bulk. ${ }^{28,29,48}$ We approximately reproduced such electron kinetic energies and EALs in additional non-resonant surface- and bulk-sensitive core-level photoelectron spectra by appropriately tuning the ionizing photon energies. Predominantly bulk solution probing depths of $\sim 4 \mathrm{~nm}$ were assured in our valence RPES experiments by the high photon energies, and therefore electron emission energies, associated with the core-to-virtual valence energy gaps (see below).

In order to compare solute and solvent signals, $0.05 \mathrm{M}$ $\mathrm{NaCl}_{(\text {aq.) }}$ solutions (Aldrich, $\geq 99 \% \mathrm{NaCl}$ crystals dissolved in millipure water) were used to produce (nearly pure) water reference samples with the required conductivity to mitigate liquid jet streaming potentials ${ }^{17,49}$ and X-ray-pulse-induced charging effects. ${ }^{50}$

Mn $\mathrm{L}_{\mathrm{II}, \mathrm{III}}$-pre-edge (Mn 2p to virtual-valence excitation) RPES measurements were performed by scanning the photon energy between 641.8-661.9 eV (with $0.15 \mathrm{eV}$ photon energy steps) and collecting electrons associated with the outer-valence-band $\mathrm{BE}$ range, 5-42 eV. Similarly, O K-pre-edge (O 1s to virtual-valence excitation) RPES measurements were performed by scanning over the 526.4-537.4 eV photon energy range (with $0.1 \mathrm{eV}$ photon energy steps) and collecting electrons within the 6-40 eV outer-valence BE range. We integrated the spectral intensities over the different $\mathrm{BE}$ ranges and plotted the results as a function of excitation photon energy to produce partial electron yield (PEY) proxies of X-ray absorption spectroscopy (XAS) spectra. From here onwards we refer to these data as PEYXAS spectra. Additional photoelectron spectra were recorded over extended BE ranges at select off-resonant and resonant photon energies to isolate resonant-signal-enhancements with high signal-to-noise ratios and span the complete $\mathrm{MnO}_{4}{ }^{-}$(aq.) (inner- and outer) valence region.

\section{Data treatment}

Shirley baselines ${ }^{51}$ were fit and subtracted from each photoelectron spectrum. Multiple baselines were generally sequentially subtracted, with each baseline covering a specific BE range. This was particularly so in the spectra extending over the entire valence band, where three baseline fits were implemented to include the outer $(\sim 5-20 \mathrm{eV}$ and $\sim 20-40 \mathrm{eV})$ and inner $(\sim 40-90 \mathrm{eV})$ valence regions. Although an imperfect 
solution for aqueous-phase spectra, the Shirley background may be judiciously applied over relatively narrow energy ranges. Generally, consistent results were obtained when applying alternative background treatment procedures, for example subtracting cubic baselines from each of the above mentioned spectral ranges. Non-resonant and resonant signals were fit using sets of Voigt functions. For valence signals for which the Lorentzian component of the Voigt fits and life-time broadening effects were confirmed to be negligible, more robust Gaussian fits were adopted and the latter fit results are reported instead. We adopt the common procedure of calibrating the valence photoelectron $\mathrm{BE}$ scales using the peak centres extracted from fits to the liquid water lowest energy ionisation peak, $1 \mathrm{~b}_{1(1)}{ }^{-1}$, with an assigned BE of $11.31 \pm 0.04 \mathrm{eV} . \ddagger^{49}$ For the core-level BE data, we utilise the similarly commonly adopted procedure of calibrating the photoelectron $\mathrm{BE}$ scale using the peak centres extracted from fits to liquid water $1 \mathrm{a}_{1}^{-1}\left(\mathrm{O} 1 \mathrm{~s}^{-1}\right)$ ionisation peaks with an assigned $\mathrm{BE}$ of $538.1 \pm$ $0.1 \mathrm{eV}^{52}$ In the case of the $1 \mathrm{t}_{2}$ (Mn 2p) spectra and solute feature BE calibrations, $1 \mathrm{a}_{1}(\mathrm{O} 1 \mathrm{~s})$ spectra were recorded under identical conditions to enable energetic referencing. For the RPES data, the centre of the water $1 b_{1(1)}$ peak in the lower photon energy off-resonant spectra was used to calibrate the $\mathrm{BE}$ scale of the related resonant spectra and RPES maps. Furthermore, associated resonant and off-resonant spectra were $\mathrm{BE}$ smoothed (5-point adjacent-averaging) with the resulting offresonant signals being subtracted from the resonant data in order to isolate resonant enhancements. (Given that the resonant and off-resonant spectra were recorded using a $100 \mathrm{meV}$ BE step size, a 5-point adjacent-average smoothing resulted in a $500 \mathrm{meV}$ effective smoothing/averaging range. Due to the generally $\geq 1 \mathrm{eV}$ spectral widths of aqueous-phase photoelectron spectroscopy features, such smoothing was found to have a negligible effect on the extracted BEs but notably enhanced our ability to highlight multi-component solute features.) We note that, for resonant spectra recorded near the $\mathrm{Mn}_{\mathrm{II}, \mathrm{II}}$ - preedges - and the associated off-resonant data - care was taken to ensure that the spectral region close to $\sim 20 \mathrm{eV}$ BE remained positive after the background correction procedure. This resulted in more consistent results overall, particularly between data treatments adopting different baseline subtraction methods. $\mathrm{MnO}_{4}^{-}{ }_{\text {(aq.) }}$ BEs and peak widths at FWHM were determined from average values extracted from fits to different data sets, with the associated errors corresponding to standard deviations. Fitting errors and the uncertainty in the water $1 b_{1(1)} B E$ value used to calibrate the BE scale were considered individually for each data set to determine the errors. A cubic baseline was fit and subtracted from the Mn L-pre-edge PEYXAS data in order to precisely extract associated resonant photon energies. In both the $\mathrm{O} \mathrm{K}$ - and Mn L-pre-edge PEYXAS data sets, the peaks associated with different resonances

\$ We choose to reference our data to this more recent value, rather than the originally determined $11.16 \pm 0.04 \mathrm{eV} .{ }^{60}$ Note that current experiments are not able to directly access absolute solution-phase binding energies, hence, requiring the adoption of an energetic reference; see discussion in ref. 59 for details. were fit using a set of Gaussian functions. In the $\mathrm{Mn} \mathrm{L}_{\mathrm{II}, \mathrm{III}}$-preedge experiments, the photon energy was calibrated using Voigt profile fits to O 1s XPS spectra recorded with the beamline fundamental and weak second harmonic. The associated electron KE differences precisely defined the implemented photon energies. The $\mathrm{O} 1 \mathrm{~s}-4 \mathrm{a}_{1}$ resonance in liquid water at $535.0 \mathrm{eV}^{53}$ was used to calibrate the photon energy in the O 1s RPES measurements.

\section{Results and discussion}

\section{Molecular structure of $\mathrm{MnO}_{4}^{-}$(aq.)}

To assign the PES features described throughout this Section, we first consider the nuclear geometric symmetry of the $\mathrm{MnO}_{4}{ }^{-}$ anion in aqueous solution. Existing theoretical calculations highlight the retention of an average tetrahedral anion symmetry in moving from the gas ${ }^{54}$ to the (bulk) aqueous-phase. $\S$ Accordingly, a $T_{\mathrm{d}}$ point group is utilised along with existing (isolated-molecule) molecular orbital diagrams ${ }^{54-56}$ to assign and label the $\mathrm{MnO}_{4}{ }^{-}$(aq.) PES signals. We assume here (and validate below) that the same symmetry assignment holds (on average) for aqueous-phase $\mathrm{MnO}_{4}{ }^{-}$both in the aqueous bulk and at the solution-gas interface. We note that the average Mn-O bond lengths (162 pm and $163 \mathrm{pm}$, respectively $)^{57}$ and the breathing vibrational mode frequencies $\left(\nu_{1} \sim 104 \mathrm{meV}\right)$ and anharmonicity constants of permanganate ${ }^{58}$ were found to be near identical in $\mathrm{KMnO}_{4}$ aqueous solutions and in the crystalline solid. Collectively, these results imply that the permanganate ion retains a similar, tetrahedral geometry in vacuum, as a component of a solid, and - most importantly in this case - in bulk aqueous solution.

Based on the above and our PES-based determination of the aqueous $\mathrm{MnO}_{4}{ }^{-}$(aq.) ionisation energetics described below, a valence and core-level molecular orbital diagram is presented for the aqueous sodium permanganate system in Fig. 1. The associated orbital energies are scaled in accordance with the average BEs extracted from our cumulative data and reported in the following sections. Hence, Fig. 1 provides a simplified schematic of the overall system energetics. We note, however, that such a single-configurational depiction of the permanganate anion electronic structure is of qualitative use only. ${ }^{54}$

Permanganate anion molecular orbitals are shown in purple in Fig. 1, with the corresponding aqueous solvent and sodium cation orbitals shown in light blue and grey, respectively. (A splitting of the $\mathrm{H}_{2} \mathrm{O}_{(1)} 3 \mathrm{a}_{1}$ molecular orbital due to intermolecular anti-bonding and bonding contributions, respectively $3 \mathrm{a}_{1-\mathrm{L}}$ and $3 \mathrm{a}_{1-\mathrm{H}},{ }^{59}$ is indicated by a light-blue fill). The positions of the $\mathrm{MnO}_{4}^{-}$(aq.) virtual valence orbitals (2e and $5 \mathrm{t}_{2}$ ) were determined through fits to $\mathrm{Na} / \mathrm{KMnO}_{4 \text { (aq.) }}$ UV-visible absorption spectra and are consistent with our RPES and PEY-XAS results (see ESI, $\dagger$ Fig. S1A). We depict the

$\S$ Established by comparison of the isolated molecule geometries in ref. 54 with the optimised aqueous-phase nuclear geometries associated with ref. 87 which were kindly shared with us during the drafting of this manuscript [JMH Olsen, Personal Communication, 09.04.2019]. 


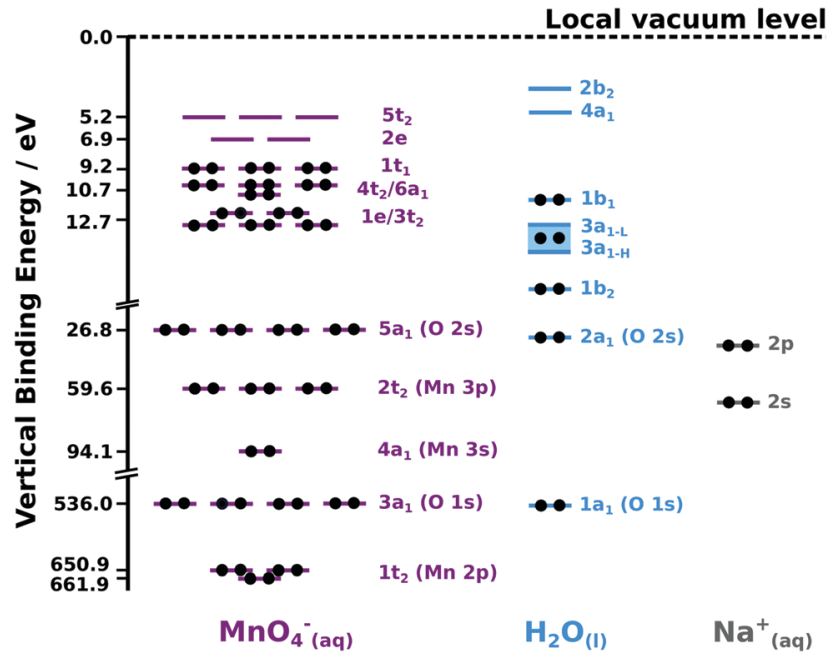

Fig. 1 Experimentally determined, binding-energy-scaled molecular orbital diagram of the aqueous $\mathrm{NaMnO}_{4}$ solution depicting the predominant electronic ground state configurations of the associated solution components. orbital occupations are denoted by black dots. (left) $\mathrm{MnO}_{4}{ }^{-}$(aq) orbitals represented by purple bars. the $1 \mathrm{t}_{2}(\mathrm{Mn} 2 \mathrm{p})$ and $3 \mathrm{a}_{1}(\mathrm{O} 1 \mathrm{~s})$ core; $4 \mathrm{a}_{1}(\mathrm{Mn} 3 \mathrm{~s})$, $2 t_{2}(M n 3 p)$, and $5 a_{1}(O 2 s)$ inner valence; $3 t_{2}, 1 e, 4 t_{2}, 6 a_{1}$, and $1 t_{1}$ outer valence; and $2 \mathrm{e}$ and $5 \mathrm{t}_{2}$ unoccupied or virtual valence orbital $\mathrm{BEs}$ are exclusively labelled on the ordinate. The $\mathrm{MnO}_{4}{ }^{-}$(aq) diagram component is adapted from the isolated-molecule diagrams reported in ref. 40 and 54 . (right) associated molecular orbital diagrams for the aqueous solvent (light blue bars and fills) and $\mathrm{Na}^{+}{ }_{(\mathrm{aq})}$ counter ion (grey bars). These diagrams are based on fits to the data reported here and in ref. 49 and 60.

predominant permanganate electronic ground state orbital occupancies $^{40}$ using black circles in Fig. 1. The ionisation features discussed in the following sections are labelled in accordance with the Fig. 1 molecular orbital labels.

The predominant electronic ground state occupancies of the chemically active outer-valence and two lowest energy virtualvalence molecular orbitals of $\mathrm{MnO}_{4}{ }^{-}$are qualitatively described by a $\left(3 \mathrm{t}_{2}\right)^{6}(1 \mathrm{e})^{4}\left(6 \mathrm{a}_{1}\right)^{2}\left(4 \mathrm{t}_{2}\right)^{6}\left(1 \mathrm{t}_{1}\right)^{6}(2 \mathrm{e})^{0}\left(5 \mathrm{t}_{2}\right)^{0}$ configuration. $^{40,56}$ These molecular orbitals are linear combinations of the frontier Mn 3d, 4s, and $4 \mathrm{p}$ and $\mathrm{O} 2 \mathrm{p}$ atomic orbitals. ${ }^{40}$ The $3 \mathrm{t}_{2}$ and 1 e features are primarily attributed to $\mathrm{Mn} 3 \mathrm{~d}$ and $\mathrm{O} 2 \mathrm{p}$ metalligand bonding interactions. The $6 \mathrm{a}_{1}$ feature is principally ascribed to $\mathrm{Mn} 4 \mathrm{~s}$ and $3 \mathrm{p}$ and $\mathrm{O} 2 \mathrm{p}_{\sigma}$ bonding interactions. The $4 \mathrm{t}_{2}$ and $1 \mathrm{t}_{1}$ orbitals are essentially O-localized, Mn-O nonbonding orbitals, with respective degrees of $\mathrm{O}-\mathrm{O}$ ligand bonding and anti-bonding character. The virtual valence $2 \mathrm{e}$ and $5 \mathrm{t}_{2}$ orbitals are the Mn-O anti-bonding analogues of the $3 t_{2}$ and 1e bonding features. The experimentally-determined valence BEs of the aqueous solvent (light blue lines) and $\mathrm{Na}^{+}$counter ion (light grey lines) orbitals are also shown in Fig. 1, to facilitate direct comparison to the anion energetics.

The experimentally-determined BEs of the majority of the solute and aqueous solvent core-levels are additionally shown in Fig. 1. The O-centred permanganate anion $3 \mathrm{a}_{1}$ solute and water $1 \mathrm{a}_{1}$ solvent orbitals have similar BEs, both corresponding to removal of $\mathrm{O}$ 1s core/inner shell electrons. The Mn-centred and spin-orbit-split $1 \mathrm{t}_{2}$ orbitals of the anion are also shown, corresponding to removal of Mn 2p core/inner-shell electrons.

\section{Direct (non-resonant) photoelectron spectra}

This section presents a subset of the experimental spectra used to nearly fully map the electronic structure of $\mathrm{MnO}_{4}{ }^{-}$(aq.) solutions and to produce - and then move beyond - the simplified schematic shown in Fig. 1. This includes identification of electronic structure fingerprints arising from the solute as well as those arising from electronic interactions between the aqueous anionic solute and solvent molecules.

Fig. 2 shows $0.2 \mathrm{M} \mathrm{NaMnO}_{4 \text { (aq.) }}$ solution XPS spectra (purple curves) measured at $635 \mathrm{eV}$ (panels A and B), $1135 \mathrm{eV}$ (panel C), and $1251 \mathrm{eV}$ (panel D) photon energies; resulting in aqueous bulk spectral sensitivities. Reference spectra from $0.05 \mathrm{M} \mathrm{NaCl}_{(\mathrm{aq} .)}$ solutions, measured under similar conditions, are also shown to highlight the aqueous solvent signals (light blue curves). Multicomponent Voigt and/or Gaussian profile fits to the data were performed to model the cumulative spectral profiles (see the ESI, $\dagger$ Fig. S2). Furthermore, as an independent and additional analysis, the baseline subtracted and intensity normalised solvent data was subtracted from the corresponding $\mathrm{NaMnO}_{4 \text { (aq.) }}$ solution data to produce difference spectra (red curves). For the spectra shown in Panel A, 3-point adjacent-averaging was applied to the solution and solvent spectra before subtraction. (Here, the solution and solvent spectra are presented with a $150 \mathrm{meV} \mathrm{BE}$ step size. Hence, a 3-point adjacent-average smoothing resulted in a $450 \mathrm{meV}$ effective smoothing/averaging range, similar to the RPES difference spectra.) The results of Gaussian or Voigt profile fits to the difference spectra peaks are additionally shown in the various panels of Fig. 2 (purple and grey fits highlight anion and cation peaks, respectively). The relatively weak inner and outer valence features in panels $\mathrm{A}$ and $\mathrm{B}$ were found - with the exception of peak 6 - to be essentially unaffected by life-time broadening effects, with the Voigt and Gaussian profile fits yielding near-identical Gaussian peak widths. There, Gaussian fits were found to be more robust than their Voigt profile equivalents and were accordingly adopted. Cumulative, multipeak Voigt fits to the BE-calibrated, baseline-corrected data and Gaussian and Voigt fit combinations to the solution-solvent difference spectra were found to yield similar BE results for all peaks. All $\mathrm{MnO}_{4}{ }^{-}$(aq.) solute features have been assigned, with the remaining signals in Fig. 2 associated with the water solvent and $\mathrm{Na}^{+}$(aq.) solute counter-ion contributions. The latter peaks have been assigned in accordance with ref. 60 and 61, respectively.

In the valence spectral regions shown in Fig. $2 \mathrm{~A}$ and $\mathrm{B}$, four distinct $\mathrm{MnO}_{4}{ }^{-}$(aq.) signals are readily extracted at $9.22 \pm$ $0.08 \mathrm{eV}$ (peak 1, panel A, right inset), 26.82 $\pm 0.04 \mathrm{eV}$ (peak 4, panel A, left inset), $59.6 \pm 0.1 \mathrm{eV}$ (peak 5, panel B), and $94.1 \pm$ $0.1 \mathrm{eV}$ (peak 6, panel B) BEs. Within the adopted molecular orbital framework, peak 1 is attributed to a $\left(1 \mathrm{t}_{1}\right)^{-1}$ ionization process and corresponds to the removal of an electron from the highest occupied molecular orbital (HOMO) and first vertical ionization energy (VIE) of $\mathrm{MnO}_{4}{ }^{-}$(aq.) . The $\mathrm{MnO}_{4}{ }^{-}$(aq.) peak 4 signal is assigned to a $\left(5 \mathrm{a}_{1}\right)^{-1}$ process, with peak 5 assigned as $\left(2 \mathrm{t}_{2}\right)^{-1}$ ionization, and peak 6 assigned as a $\left(4 \mathrm{a}_{1}\right)^{-1}$ process. The latter three features are thought to be predominantly associated with ionisation of the ligand $\mathrm{O} 2 \mathrm{~s}, \mathrm{Mn} 3 \mathrm{p}$, and $\mathrm{Mn}$ $3 \mathrm{~s}$ atomic orbitals, respectively. ${ }^{41,62}$ However, as will be shown 

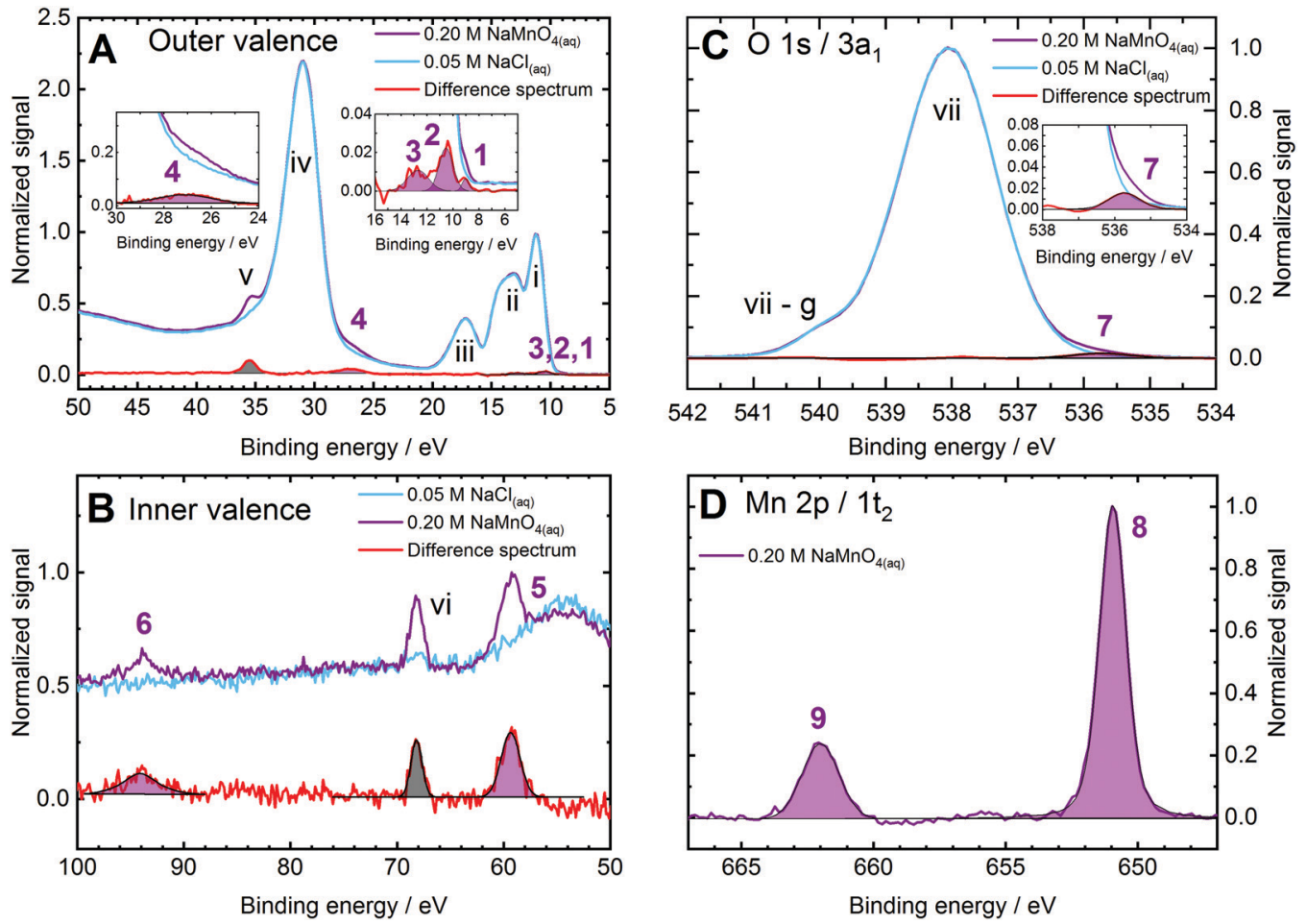

Fig. 2 Bulk-sensitive $\mathrm{X}$-ray photoelectron spectra of $0.2 \mathrm{M}$ aqueous $\mathrm{NaMnO}_{4}$ (purple curves) and $0.05 \mathrm{M}$ sodium chloride (blue curves, representative of the aqueous solvent) solutions recorded at the P04 beamline, PETRA III (panels a, c, and d) and at the U49/2 PGM-1 beamline, BESSY II (panel b). Solution-solvent difference spectra (red curves) are plotted in panels (a)-(c) to highlight solute features. Gaussian (panel a and peaks 5 and vi in panel b) or Voigt (peak 6 in panel b and all features in panels $c$ and d) profile fits to the solute anion and cation signals are illustrated by purple and grey fills, respectively. (a) Valence band and solution-solvent difference spectra recorded at a $635 \mathrm{eV}$ photon energy. The right inlay shows the $\mathrm{MnO}_{4}{ }^{-}$(aq.) lowest energy ionizing transition features (peaks $1\left(1 \mathrm{t}_{1}^{-1}\right), 2\left(4 \mathrm{t}_{2}{ }^{-1} / 6 \mathrm{a}_{1}{ }^{-1}\right)$, and $\left.3\left(1 \mathrm{e}^{-1} / 3 \mathrm{t}_{2}{ }^{-1}\right)\right)$ while the left inlay shows an oxygen-centred inner valence ionization feature (peak $4\left(5 \mathrm{a}_{1}^{-1}\right)$ ). (b) As for panel a with a focus on a higher binding energy range and highlighting additional $\mathrm{MnO}_{4}{ }^{-}$(aq). inner valence features (peak $5\left(2 \mathrm{t}_{2}{ }^{-1}\right)$ and $\left.6\left(4 \mathrm{a}_{1}{ }^{-1}\right)\right)$. (c) Oxygen 1s core ionization and difference spectra recorded at a photon energy of $1135 \mathrm{ev}$, with the $\mathrm{MnO}_{4}^{-}$(aq.) Oxygen-ligandassociated feature (peak $7\left(3 \mathrm{a}_{1}{ }^{-1}\right)$ ) highlighted in the panel inlay. (d) Manganese $2 \mathrm{p}, \mathrm{MnO}_{4}{ }^{-}$(aq.) core ionization spectrum recorded at a photon energy of $1251 \mathrm{eV}$ and highlighting the spin-orbit split $\mathrm{Mn}$ (vii) solute metal centre features (peaks 8 and $9\left(1 \mathrm{t}_{2}{ }^{-1}\right)$ ). the $\mathrm{MnO}_{4}{ }^{-}$(aq.) $\mathrm{BEs}$ extracted from this figure and similar data sets are summarized in Table 1. The solvent and counter-ion $\mathrm{Na}^{+}$ionization features are labelled with lower-case roman numerals and the associated binding energies are summarized in Table 2 .

in the following Resonant photoemission spectra section, these inner-valence orbital features are found to be hybridized.

Upon performing cumulative Gaussian fits to the solutionsolvent difference spectra shown in the right inset of Fig. 2, panel A, it was possible to extract two more $\mathrm{MnO}_{4}{ }^{-}$(aq.) valence BEs: $10.67 \pm 0.04 \mathrm{eV}$ and $12.84 \pm 0.09 \mathrm{eV}$ (peaks 2 and 3). Based on Fig. 1 and associated ref. 40 and 54 these features are tentatively assigned to overlapping $\left(4 \mathrm{t}_{2}\right)^{-1} /\left(6 \mathrm{a}_{1}\right)^{-1}$ and $(1 \mathrm{e})^{-1} /$ $\left(3 \mathrm{t}_{2}\right)^{-1}$ ionizing transitions, respectively. The assignments of these peaks to multiple final states is supported by their relatively large feature widths (see Table 1 for details); as well as the identification of narrower but spectrally overlapping resonantly enhanced ionisation features that will be discussed in the following Resonant photoemission spectra section.

While peak 2 is reproducibly extracted from bulk- (and surface-sensitive) difference spectra data, the higher BE peak 3 feature directly underlies the gas-phase water $1 b_{1}$ (HOMO) ionization vibronic peaks (that are generally and variably perturbed by liquid sample electrokinetic/ionization-induced charging). Accordingly, the $\sim 12.8 \mathrm{eV}$ BE feature (peak 3) could only be isolated when tight vertical X-ray focusing conditions were implemented (as afforded at the P04 beamline) that minimized gas-phase spectral contributions. When larger vertical X-ray focal spot sizes and/or lower ionizing photon energies were implemented, this peak was superimposed on large gas-phase signals and could not be extracted (see ESI, $\dagger$ Fig. S3, for example). Hence, we additionally rely on our RPES experiments, reported in the subsequent section, to confirm this BE.

$\mathrm{MnO}_{4}{ }^{-}$(aq.) solution O 1s $\left(3 \mathrm{a}_{1}\right)$ and $\mathrm{Mn} 2 \mathrm{p}\left(1 \mathrm{t}_{2}\right)$, as well as aqueous solvent reference $O 1 \mathrm{~s}\left(1 \mathrm{a}_{1}\right)$, core-level XPS spectra were additionally recorded. Exemplary data, a solution-solvent $\mathrm{O} 1 \mathrm{~s}$ difference spectrum, and associated Voigt fits to the data are shown in Fig. 2 panels $\mathrm{C}$ and D. Here the solute oxygenligand feature - peak 7 at $536.0 \pm 0.1 \mathrm{eV},\left(3 \mathrm{a}_{1}\right)^{-1}$ - is uniquely and reproducibly extracted and found to be shifted to lower BEs with respect to the $\mathrm{O} 1 \mathrm{~s},\left(1 \mathrm{a}_{1}\right)^{-1}$, feature of the aqueous solvent by $2.1 \mathrm{eV}$. (Note that peak vii-g is associated with the gas-phase water $\mathrm{O} 1 \mathrm{~s}$ contribution which is differentially shifted between the sample solution and aqueous reference spectra due to changes in electrokinetic and ionization-induced charging effects.) The solute-only Mn $2 \mathrm{p}_{3 / 2}$ and $\mathrm{Mn} 2 \mathrm{p}_{1 / 2}$ features - peaks 8 and 9 at $650.9 \pm 0.1 \mathrm{eV}$ and $661.9 \pm 0.1 \mathrm{eV}$, respectively, 
Table 1 Summary of the $\mathrm{MnO}_{4}{ }^{-}$(aq.) solute BEs and associated Gaussian peak width components, FWHM $M_{G}$ extracted from the direct PES experiments (top panel) and RPES experiments (bottom panel). The listed $\mathrm{FWHM}_{\mathrm{G}}$ values were extracted from data sets recorded at $280 \pm 3 \mathrm{~K}$. The error bounds correspond to the standard deviations of the results extracted from different data sets. $F_{W H M}$ values were directly extracted from Gaussian fits in all cases except for peaks 6-9 (top) and 3-a (bottom). In the latter cases, Voigt profile fits to the data revealed non-negligible lifetime broadening effects. We accordingly selectively report the Gaussian width components of the Voigt profile fits. Hence, all reported peak widths cover the inhomogeneous broadening associated with the aqueous environment and the limits of our experimental energy resolution but exclude lifetime broadening effects. Concerning peak 6 , due to its low intensity and spectral breadth, we supplement the peak width extracted from $0.2 \mathrm{M}$ solutions with the result from fits to higher signal-to-noise ratio $0.75 \mathrm{M} \mathrm{NaMnO}_{4 \text { (aq) }}$ solution data, the latter is reported in parenthesis

\begin{tabular}{lcll}
\hline Label & Peak origin & $\mathrm{BE} / \mathrm{eV}$ & $\mathrm{FWHM}_{\mathrm{G}} / \mathrm{eV}$ \\
\hline $\mathrm{MnO}_{4}{ }^{-}$(aq.) & $-\mathrm{XPS}$ & & \\
1 & $\left(1 \mathrm{t}_{1}\right)^{-1}$ & $9.22 \pm 0.08$ & $0.8 \pm 0.1$ \\
2 & $\left(4 \mathrm{t}_{2} / 6 \mathrm{a}_{1}\right)^{-1}$ & $10.67 \pm 0.04$ & $1.2 \pm 0.1$ \\
3 & $\left(1 \mathrm{e} / 3 \mathrm{t}_{2}\right)^{-1}$ & $12.84 \pm 0.09$ & $1.9 \pm 0.2$ \\
4 & $\left(5 \mathrm{a}_{1}\right)^{-1}$ & $26.82 \pm 0.04$ & $2.1 \pm 0.6$ \\
5 & $\left(2 \mathrm{t}_{2}\right)^{-1}$ & $59.6 \pm 0.1$ & $2.3 \pm 0.2$ \\
6 & $\left(4 \mathrm{a}_{1}\right)^{-1}$ & $94.1 \pm 0.1$ & $2 \pm 4(2.70 \pm 0.07)^{*}$ \\
7 & $\left(3 \mathrm{a}_{1}\right)^{-1}$ & $536.0 \pm 0.1$ & $0.9 \pm 0.3$ \\
8 & $\left(1 \mathrm{t}_{2}\right)^{-1}$ & $650.9 \pm 0.1$ & $0.93 \pm 0.06$ \\
9 & $\left(1 \mathrm{t}_{2}\right)^{-1}$ & $661.9 \pm 0.1$ & $1.0 \pm 0.9$ \\
& & & \\
$\mathrm{MnO}_{4}{ }^{-}{ }^{\text {(aq.) }}$ & $-\mathrm{RPES}$ participator Auger $_{2-0}$ \\
$2-\mathrm{a}$ & $\left(4 \mathrm{t}_{2}\right)^{-1}$ & $9.85 \pm 0.08$ & $0.7 \pm 0.2$ \\
$3-\mathrm{a}$ & $\left(1 \mathrm{e} \text { or } 3 \mathrm{t}_{2}\right)^{-1}$ & $12.66 \pm 0.04$ & $0.83 \pm 0.04$ \\
5 & $\left(2 \mathrm{t}_{2}\right)^{-1}$ & $59.8 \pm 0.4$ & $2.6 \pm 0.6$ \\
& &
\end{tabular}

$\left(1 \mathrm{t}_{2}\right)^{-1}$ - are found to be shifted to higher BEs by $\sim 12 \mathrm{eV}$ with respect to metallic $\mathrm{Mn}^{37,63,64}$ The large positive $\mathrm{Mn} \mathrm{2p}$ chemical shifts reflect the formal $\mathrm{Mn}^{\mathrm{VII}+}$ oxidation state of the $\mathrm{MnO}_{4}^{-}{ }^{-}$(aq.) metal centre and its covalent bonding to the $\mathrm{O}$ ligands. While the $\mathrm{MnO}_{4}{ }^{-}$(aq.) and $\mathrm{H}_{2} \mathrm{O}_{(\mathrm{l})}$ oxygen atoms share the same formal $\mathrm{O}^{\mathrm{II}-}$ oxidation state, the respective negative chemical shift of the solute peak indicates a higher $\mathrm{MnO}_{4}^{-}$(aq.) O-ligand charge density. This charge excess may be attributed to intramolecular (ligand-ligand) and potential intermolecular (ligand-water) $\mathrm{O}-\mathrm{O}$ covalent bonding interactions.

BEs and peak FWHMs of all $\mathrm{MnO}_{4}^{-}{ }_{\text {(aq.) }}$ associated signals were extracted from fits to multiple bulk-sensitive data sets, similar to that shown in Fig. 2. The averaged results and their standard deviations, are summarised in the top part of Table 1. Gaussian peak FWHMs are specifically reported for liquid jet temperatures of $280 \pm 3 \mathrm{~K}$. The BE values listed on the ordinate of Fig. 1 correspond to the values summarised in Table 1 . Corresponding averaged results from the BE peak fits to the water and $\mathrm{Na}^{+}$(aq.) spectral contributions to Fig. 2 and similar data sets are also illustrated in Fig. 1. These solvent and cation peak fit results are additionally summarized in Table 2.

Solution-gas interface and counter ion effects. To explore whether the electronic structure of $0.2 \mathrm{M} \mathrm{MnO}_{4}{ }^{-}$(aq.) solutions are different at the solution-gas interface compared to the bulk liquid, we also measured valence PES spectra with significantly lower ionising photon energies. ESI, $\dagger$ Fig. S3 and Table S1 respectively show analogous data to that shown in Fig. 2 and
Table 1, in this case recorded with $150 \mathrm{eV}$ (outer and inner valence, panel A), $688 \mathrm{eV}\left(4 \mathrm{a}_{1} / \mathrm{O}\right.$ 1s core, panel B), and $800 \mathrm{eV}\left(1 \mathrm{t}_{2} / \mathrm{Mn} \mathrm{2p}\right.$ core, panel C) photon energies. These measurements allowed us to selectively probe the solutiongas-phase interface. The surface-sensitive data are found to be very similar to the bulk-sensitive data, except for the different relative intensities of most of the peaks. This largely reflects the changes of relative ionisation cross sections for the different photon energies, although smaller effects due to photoelectron elastic scattering occur as well. The most important observation from Fig. S3 (ESI $\dagger$ ) is that the BEs of all features are unaffected as we decrease our sensitivity to the aqueous bulk of the solution, at least within the limits of our experimental uncertainties and for solution concentrations $\leq 0.2 \mathrm{M}$ (see ESI, $†$ Table S1). With this in mind, the BEs, FWHMs, and associated errors extracted from the surface-sensitive data shown in Fig. S3 (ESI $\dagger$ ) are also represented in the top section of Table 1 , as well as the schematic BE summary shown on the ordinate of Fig. 1. From these results, we conclude that potential incomplete hydration at the interface has too small an effect to be detected in our experiments. This provides the basis for our aforementioned assumption that (on average) a similar permanganate molecular structure exists both in the aqueous bulk and at the solution-gas interface.

Exceptionally, one additional feature is extracted in the surface-sensitive $3 \mathrm{a}_{1}{ }^{-1}$ (O $\left.1 \mathrm{~s}\right)$ data that is not discernible in the bulk-sensitive experiments (see ESI, $\dagger$ Fig. S3B and S5B). We note either a slight increase in the width of peak vii in the solution spectra occurs with respect to the solvent reference spectra or that an additional ionisation feature emerges at a $\mathrm{BE}$ of $538.3 \pm 0.2 \mathrm{eV}$ (unfortunately, these two spectral forms cannot be differentiated due to the overlap of peak vii with its gas-phase counterpart, vii-g, on the high $\mathrm{BE}$ side of the $3 \mathrm{a}_{1}{ }^{-1} / \mathrm{O} 1 \mathrm{~s}$ peak). Notably, the BE of this feature - marked with $\mathrm{a} \dagger-$ can only be extracted from the difference spectra, with cumulative Voigt profile fits to the solution data yielding a single solute contribution to the $3 \mathrm{a}_{1}{ }^{-1}$ (O 1s) spectrum. This broadening of the solvent feature or additional solute peak may be attributable to a broader range of hydration environments

Table 2 As in the top panel of Table 1 but showing the $\mathrm{H}_{2} \mathrm{O}_{(1)}$ (top panel) and $\mathrm{Na}^{+}{ }_{\text {(aq.) }}$ (bottom panel) BEs and FWHM peak widths extracted from cumulative Gaussian (valence-band) and Voigt profile (O 1s) fits to the direct PES spectra. The $\mathrm{Na}^{+}{ }_{\text {(aq.) }} 2 \mathrm{p} \mathrm{BE}$ was extracted from solution-solvent difference spectra. Entries in bold font highlight the BE reference values used to calibrate the PES and RPES data BEs

\begin{tabular}{lllc}
\hline Label & Peak origin & BE/eV & FWHM/eV \\
\hline $\mathrm{H}_{2} \mathrm{O}_{(\mathrm{l})}-\mathrm{XPS}$ & & & \\
$\mathrm{i}$ & $\left(1 \mathrm{~b}_{1}\right)^{-1}$ & $\mathbf{1 1 . 3 1} \pm \mathbf{0 . 0 4}$ & $1.42 \pm 0.03$ \\
$\mathrm{ii}-\mathrm{a}$ & $\left(3 \mathrm{a}_{1-\mathrm{L}}\right)^{-1}$ & $13.02 \pm 0.05$ & $1.6 \pm 0.1$ \\
$\mathrm{ii}-\mathrm{b}$ & $\left(3 \mathrm{a}_{1-\mathrm{H}}\right)^{-1}$ & $14.46 \pm 0.06$ & $1.6 \pm 0.1$ \\
iii & $\left(1 \mathrm{~b}_{2}\right)^{-1}$ & $17.42 \pm 0.04$ & $2.32 \pm 0.06$ \\
iv & $\left(2 \mathrm{a}_{1}\right)^{-1}$ & $31.04 \pm 0.04$ & $2.97 \pm 0.02$ \\
vii & $\left(1 \mathrm{a}_{1}\right)^{-1}$ & $\mathbf{5 3 8 . 1} \pm \mathbf{0 . 1}$ & $1.65 \pm 0.03$ \\
& & & \\
$\mathrm{Na}^{+}{ }^{(\text {aq. })}-\mathrm{XPS}$ & & & \\
$\mathrm{v}$ & $2 \mathrm{p}$ & $35.65 \pm 0.04$ & $1.25 \pm 0.08$ \\
vi & $2 \mathrm{~S}$ & $68.46 \pm 0.04$ & $1.6 \pm 0.1$
\end{tabular}


being sampled at the aqueous-gas interface and/or a reduction of the solute molecular symmetry at the interface. We favour the former explanation due to the indiscernible effects of a potential reduction of molecular symmetry on the other surface-sensitive PES features. However, a firm assignment requires more in-depth experimental analysis (for example concentration-dependent, surface- and bulk-sensitive measurements) which is beyond the scope of the work presented here.

As a further cross-check of our measurements and results, we have additionally probed the effects of the counter-ion on the extracted BEs (see ESI, $\dagger$ Fig. S5). No BE shifts were observed in changing the counter-ion of the solution from $\mathrm{Na}^{+}$to $\mathrm{K}^{+}$at fixed $0.2 \mathrm{M}$ concentration (note that such comparisons are only valid at such low concentrations due to the aqueous solubility limit of $\mathrm{KMnO}_{4}{ }^{65}$ ). The consistency of the $\mathrm{MnO}_{4}{ }^{-}$anion BEs with counter-ion was preserved in both aqueous bulk- and interfacially-sensitive experiments, see Fig. S6, S10 and Tables S2, S5 in the ESI $\dagger$ for details. Accordingly, we attribute the solute signals reported here to separated and hydrated $\mathrm{MnO}_{4}{ }^{-}$anions and $\mathrm{Na}^{+} / \mathrm{K}^{+}$cations (in spite of previous reports regarding partial aqueous dissociation of permanganate salts ${ }^{66}$ ).

$\mathrm{MnO}_{4}{ }^{-}$electronic structure comparison across phases. We now return to Fig. 1 for a quantitative comparison of the electronic energetics of the aqueous permanganate system with the corresponding solid-state and gas-phase species. In the solid-phase $\mathrm{KMnO}_{4}$ PES measurements reported by Reinert et al. ${ }^{37}$ similar outer and inner valence BEs have been extracted as in the aqueous phase. When the associated solid-state work function $(5.3 \mathrm{eV})$ is accounted for (to bring the solid-state results onto a common local-vacuum reference level 1 ), the solid-state valence BEs are found to be between $0.0-0.4 \mathrm{eV}$ in excess of their aqueous counterparts. We attribute these shifts to decreasing degrees of dielectric screening as we move from the aqueous solution to the solid-state and/or orbital (or state) specific $\mathrm{MnO}_{4}{ }^{-}$(aq.) ${ }^{-} \mathrm{H}_{2} \mathrm{O}_{(1)}$ intermolecular bonding interactions. Considering the $\mathrm{Mn} 2 \mathrm{p}$ and $\mathrm{O}$ 1s core-level BEs determined in the solid-phase (650.8 eV and $536.2 \mathrm{eV}$ ), near-negligible aqueous versus solid BE differences of just $-0.1 \pm 0.1 \mathrm{eV}$ and $+0.2 \pm$ $0.1 \mathrm{eV}$ are extracted with respect to the vacuum level. These results highlight similarly net-screened local charge distributions across the two phases.

As expected, the gas-phase permanganate anion is significantly less stable with respect to ionisation than the aqueousand solid-phase species. The $\left(1 \mathrm{t}_{1}\right)^{-1} / \mathrm{HOMO}^{-1} \mathrm{VIE}$ of isolated $\mathrm{MnO}_{4}{ }^{-}$was determined to be $4.91 \pm 0.03 \mathrm{eV}$ (relative to vacuum) by Gutsev et al. ${ }^{34}$ with an associated AIE of $4.80 \pm$ $0.1 \mathrm{eV}$ also being extracted. The $\sim 4.3 \mathrm{eV}$ disparity between the gas- and aqueous-phase VIEs reflects both the anion stabilisation afforded by the aqueous solvent - i.e. the Gibbs free energy of hydration, $\Delta G_{\text {hyd }}^{0}$ - and the (oxidative) solvent reorganisation energy $-\lambda_{\text {ox }}$. Similar energetic offsets are observed between the gas- and aqueous-phase $\left(4 \mathrm{t}_{2}\right)^{-1} /(\mathrm{HOMO}-1)^{-1}$ VIEs $(\sim 4.5 \mathrm{eV})$. However, the inference that the second aqueous phase

T Here, the work function has been assumed to be representative of a $\mathrm{KMnO}_{4}$ crystal-face-independent value. ${ }^{88}$ ionisation feature is associated with two unresolved final states i.e. a $\left(4 \mathrm{t}_{2} / 6 \mathrm{a}_{1}\right)^{-1}$ process - prevents a direct comparison of the second PES peaks across phases. We return to this point in the subsequent Absolute energetics and thermodynamics of $\mathrm{MnO}_{4}{ }^{-}$(aq.) solutions section.

\section{Resonant photoemission spectra}

RPES data and core-hole relaxation mechanisms. To further interrogate the electronic structure of $\mathrm{MnO}_{4}{ }^{-}$(aq.) solutions, we recorded valence band PES spectra as a function of ionising photon energy in the vicinity of the $\mathrm{Mn} \mathrm{L}_{\mathrm{II} / \mathrm{III}^{-}}$and $\mathrm{O}$ K-edges using 0.2 $\mathrm{M} \mathrm{NaMnO}_{4}{ }^{-}$(aq.) solutions. Fig. 3A (left) shows an associated, exemplary $\mathrm{Mn}_{\mathrm{III}}$ pre-edge RPES map, along with a PEY-XAS spectrum (right) extracted from the map via full BE-axis-integration. A corresponding $\mathrm{Mn} \mathrm{L}_{\mathrm{II}}$ pre-edge map and spectrum is shown in the ESI $\dagger$ (see Fig. S7, panel A). An analogous RPES map (left) and spectrum (right) is shown for the O K-pre-edge region in Fig. 3D. The signal enhancements observed in the RPES maps are associated with intramolecular Auger emission and/or non-local, intermolecular relaxation processes that occur after photo-excitation of a $1 \mathrm{t}_{2}\left(\mathrm{Mn} 2 \mathrm{p}_{3 / 2}\right.$ or Mn $2 \mathrm{p}_{1 / 2}$, see Fig. $3 \mathrm{~A}$ and Fig. S7A (ESI $\dagger$ ), respectively) or $3 \mathrm{a}_{1}$ (O 1s, Fig. 3D) core electron into an unoccupied virtual valence orbital; either $2 \mathrm{e}$ - the lowest unoccupied molecular orbital, LUMO - or $5 t_{2}$ - corresponding to the LUMO+1 orbital. We assign the PEY-XAS spectral features based on the UV-visible absorption spectra energy gaps and assignments (see ESI, $\dagger$ Fig. S1), the $\mathrm{MnO}_{4}{ }^{-}$electronic structure literature, ${ }^{40,54,56}$ the solid-state results of Reinert et al., ${ }^{67}$ and expected electron correlation effect magnitudes. Through cumulative fits to the PEY-XAS spectra shown in Fig. 3A, D and Fig. S7A (ESI $\dagger$ ), the following photon energy resonances were extracted and assigned (see ESI, $\dagger$ Fig. S1B and C): $643.4 \pm 0.1 \mathrm{eV}\left(1 \mathrm{t}_{2} / \mathrm{Mn}\right.$

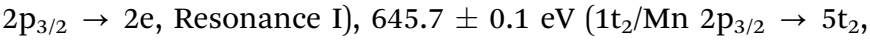
Resonance II), $654.2 \pm 0.2 \mathrm{eV}\left(1 \mathrm{t}_{2} / \mathrm{Mn} 2 \mathrm{p}_{1 / 2} \rightarrow 2 \mathrm{e}\right.$, Resonance III),

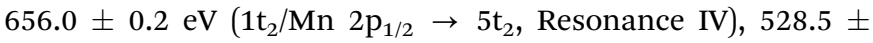
$0.1 \mathrm{eV}\left(3 \mathrm{a}_{1} / \mathrm{O} 1 \mathrm{~s} \rightarrow 2 \mathrm{e}\right.$, Resonance $\left.\mathrm{V}\right)$, and $530.0 \pm 0.1 \mathrm{eV}\left(3 \mathrm{a}_{1} /\right.$ $\mathrm{O} 1 \mathrm{~s} \rightarrow 5 \mathrm{t}_{2}$, Resonance $\left.\mathrm{VI}\right)$. These photon energies are summarised in Table 3. We emphasise that the energy gaps between the $2 \mathrm{e}$ and $5 \mathrm{t}_{2}$ virtual valence features associated with the resonance I and II, III and IV, and V and VI pairs differ significantly from each other and the gaps extracted from the UV-visible spectra. This is due to the disparate core and valence electron correlation effects associated with the different excitations. Note that the core-to-virtual valence resonances of the aqueous solvent span the 533-550 eV range ${ }^{53,68}$ and have no overlap with the $\mathrm{MnO}_{4}{ }^{-}$(aq.) resonances discussed here.

In order to probe Auger contributions from both inner and outer valence orbitals, $0.2 \mathrm{M} \mathrm{NaMnO}_{4 \text { (aq.) }}$ solution PES spectra were recorded with improved signal-to-noise ratios at each of the aforementioned resonant photon energies over an extended $\mathrm{BE}$ range. Fig. 3B and $\mathrm{C}$ show extended valence-band RPES spectra recorded at the Mn Resonances I (red curve) and II (blue curve), respectively. In each panel, a spectrum recorded at a lower, off-resonant photon energy $(638.0 \pm 0.1 \mathrm{eV})$ is additionally plotted as a non-resonant reference (black curves; note the 

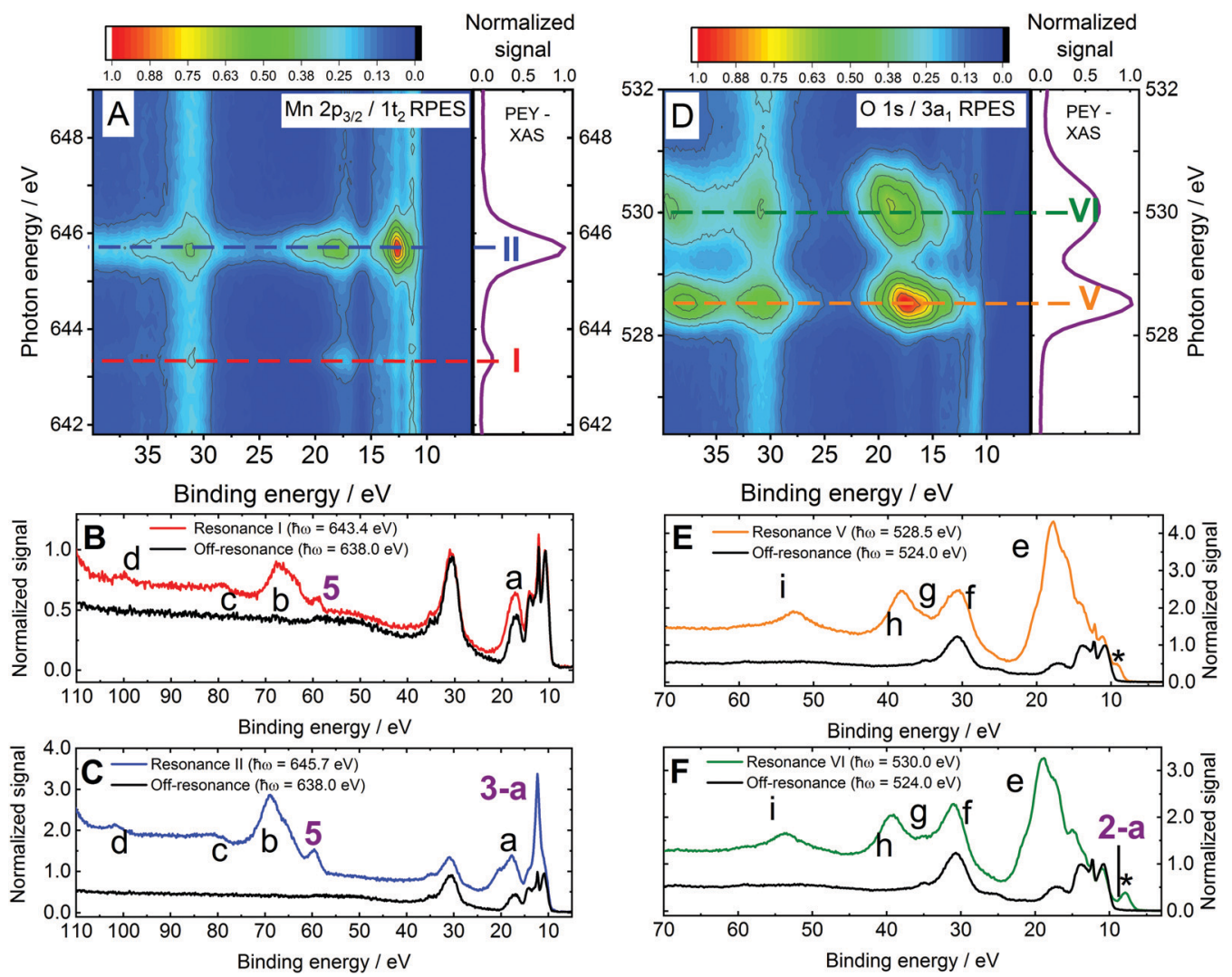

Fig. 3 RPES spectra from $0.2 \mathrm{M} \mathrm{NaMnO}_{4 \text { (aq.) }}$ solutions recorded at the U49/2 PGM-1 beamline, BESSY II. (A) $1 \mathrm{t}_{2} / \mathrm{Mn} 2 \mathrm{p}_{3 / 2}$ to virtual valence RPES map

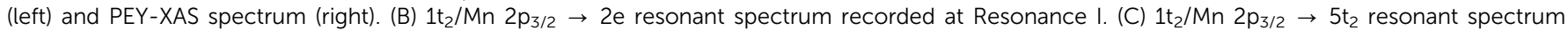
recorded at Resonance II. (D) $3 a_{1} / O$ is to virtual valence RPES map (left) and PEY-XAS spectrum (right). (E) $3 a_{1} / O 1 s \rightarrow 2 e$ resonant spectrum recorded at Resonance $\mathrm{V}$. (F) $3 \mathrm{a}_{1} / \mathrm{O} 1 \mathrm{~s} \rightarrow 5 \mathrm{t}_{2}$ resonant spectrum recorded at Resonance VI. The photon energies associated with Resonances I, II, V, and $\mathrm{VI}$ are highlighted by dashed lines in panels a and d. Off-resonant spectra are shown as reference signals (i.e. without resonant-enhancement, black curves) in panels b, c, e, and f. Participator auger signals are labelled with numbers (see the bottom panel of Table 1) while spectator auger and potential ICD signals are labelled with letters (see Table 4). The asterisks highlight an artefact signal associated with ionisation of $\mathrm{H}_{2} \mathrm{O}_{(1)} 1 a_{1} / \mathrm{O}_{1} 1 \mathrm{~s}$ electrons by the second harmonic of the beam line. Although the experiment strictly detects both the kinetic energies of the direct photoelectrons and Auger electrons, with a binding energy scale generally only having legitimate meaning for the former, we plot the abscissas on a binding energy scale here to enable ready comparison - particularly of participator auger features - with the direct XPS data presented in the previous section.

Table 3 Overview of the aqueous-phase $\mathrm{MnO}_{4}{ }^{-}$core-to-virtual valence resonant excitation energies extracted from the RPES and PEY-XAS results shown in Fig. 3 and ESI, Fig. S7

\begin{tabular}{|c|c|c|}
\hline Resonance & Transition & Photon energy/eV \\
\hline I & $1 \mathrm{t}_{2}\left(\mathrm{Mn} 2 \mathrm{p}_{3 / 2}\right) \rightarrow 2 \mathrm{e}$ & $643.4 \pm 0.1$ \\
\hline II & $1 \mathrm{t}_{2}\left(\mathrm{Mn} 2 \mathrm{p}_{3 / 2}\right) \rightarrow 5 \mathrm{t}_{2}$ & $645.7 \pm 0.1$ \\
\hline III & $1 \mathrm{t}_{2}\left(\mathrm{Mn} 2 \mathrm{p}_{1 / 2}\right) \rightarrow 2 \mathrm{e}$ & $654.2 \pm 0.2$ \\
\hline IV & $1 \mathrm{t}_{2}\left(\mathrm{Mn} \mathrm{2} \mathrm{p}_{1 / 2}\right) \rightarrow 5 \mathrm{t}_{2}$ & $656.0 \pm 0.2$ \\
\hline $\mathrm{V}$ & $3 \mathrm{a}_{1}(\mathrm{O} 1 \mathrm{~s}) \rightarrow 2 \mathrm{e}$ & $528.5 \pm 0.1$ \\
\hline VI & $3 \mathrm{a}_{1}(\mathrm{O} 1 \mathrm{~s}) \rightarrow 5 \mathrm{t}_{2}$ & $530.0 \pm 0.1$ \\
\hline
\end{tabular}

absence of resonant enhancement signals therein). Corresponding spectra associated with Mn Resonances III and IV are shown in the ESI $\dagger$ (see Fig. S7, panels B and C). Fig. 3E and $\mathrm{F}$ show analogous full valence-band spectra recorded at $\mathrm{O}$ Resonances V (orange curve) and VI (green curve), respectively. The associated off-resonant reference spectrum shown in both panels (black curves) was recorded at a $524.0 \pm 0.1 \mathrm{eV}$ photon energy. Resonantly enhanced signals are labelled in panels $\mathrm{B}$,
C, E, and F of Fig. 3, with numbers that identify solute participator Auger signals (see Fig. 4, panels $\mathrm{B}_{1} / \mathrm{C}_{1}$ ) and letters that highlight solute spectator Auger signals and potential resonant non-local decay processes (see Fig. 4, panels $\mathrm{B}_{2} / \mathrm{C}_{2}$ and $B_{3} / C_{3}$, respectively). The asterisks in panels $E$ and $F$ highlight artefact signals associated with removal of $\mathrm{H}_{2} \mathrm{O}_{(1)} \quad 1 \mathrm{a}_{1}$ (O 1s) electrons through absorption of the unfiltered second harmonic of the U49/2 PGM-1 beamline. Notably, we similarly recorded RPES data from $0.15 \mathrm{M} \mathrm{KMnO}_{4}{ }^{-}$(aq.) solutions in the vicinity of the $\mathrm{Mn} \mathrm{L}_{\mathrm{II} / \mathrm{III}}$ and $\mathrm{O}$ K-edges. Similar results were extracted with the two solutions, in all cases (compare Fig. 3 with ESI, $\dagger$ Fig. S6).

A schematic of possible processes contributing to the resonant enhancement signals associated with Fig. 3 (and the complementary ESI $\dagger$ data) is shown in Fig. 4. Fig. 4A illustrates the first step of the RPES process, the core-to-virtual-valence transition that generates a highly-excited and unstable, corehole state localised at one of the atomic constituents of the $\mathrm{MnO}_{4}{ }^{-}$(aq.) solute. The short-lived (few- to sub-femtosecond) nature of the initially photo-excited core-hole state may lead to 

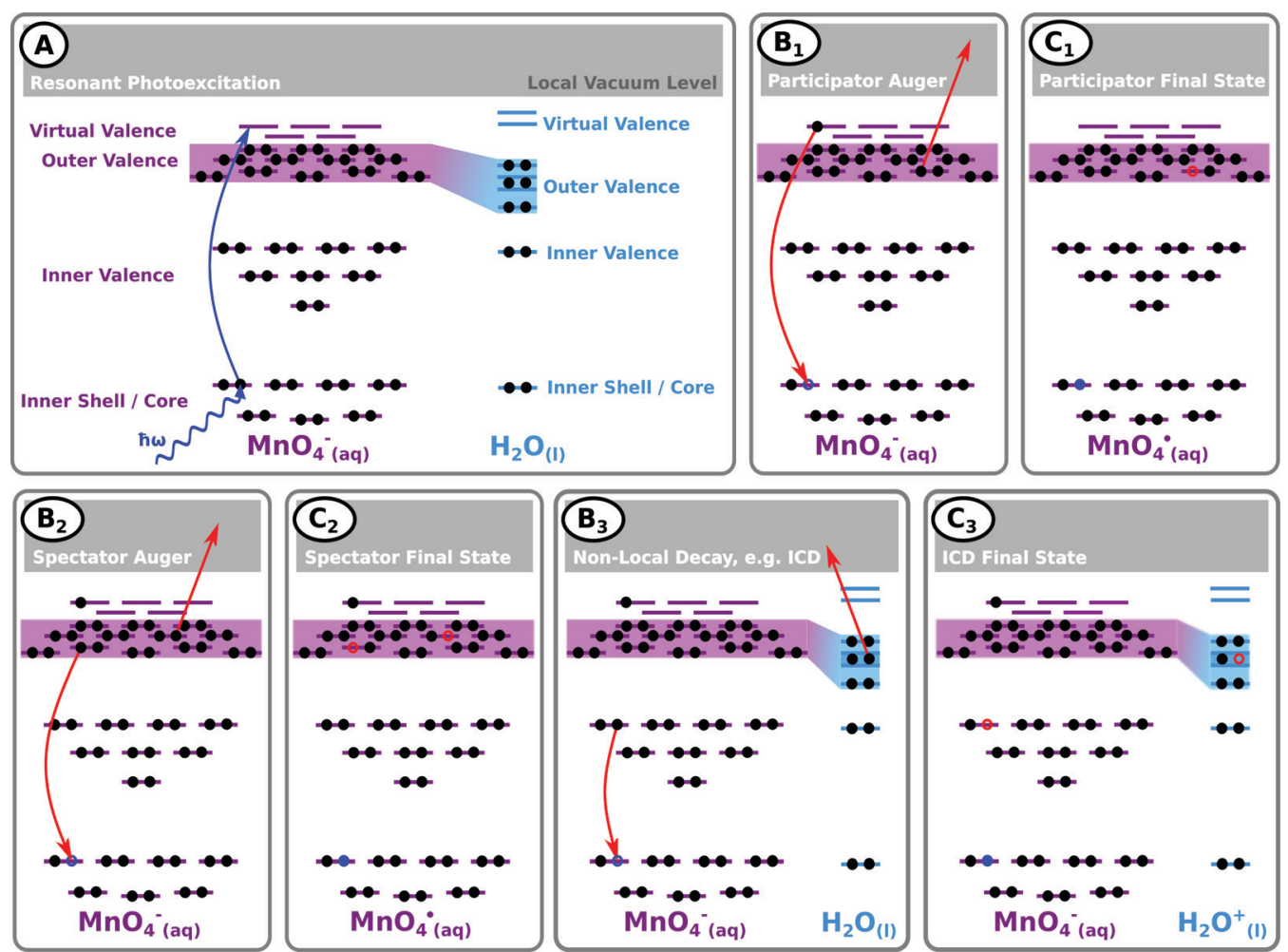

Fig. 4 Schematic of exemplary RPES processes occurring in aqueous $\mathrm{MnO}_{4}{ }^{-}$solutions. (A) Depiction of the initial resonant excitation along with an overview of the photo-excited virtual valence, chemically active and delocalised outer valence, (more) localised but environmentally-sensitive inner valence, and inner shell/core orbitals. $\left(B_{1}\right)$ Highlights solute participator resonant auger processes that result in $\left(C_{1}\right)$ singly-ionised, single-hole aqueous permanganate radical final states and permit the extraction of $\mathrm{MnO}_{4}{ }^{-}$solute ionisation signals and $\mathrm{BEs}$ that are otherwise obscured by the dominant solvent background signal. $\left(B_{2}\right)$ Highlights a solute spectator resonant auger process that generates $\left(C_{2}\right)$ electronically excited states of the aqueous permanganate radical and allows molecular orbital hybridisation and intramolecular delocalisation phenomena to be assessed. $\left(\mathrm{B}_{3}\right)$ An example of a potential non-local core-hole relaxation process, in this case resonant intermolecular coulombic decay (ICD). $\left(\mathrm{C}_{3}\right) \mathrm{ICD}$ charge-separated, electronically excited $\mathrm{MnO}_{4}^{-}$(aq.) anion and highly unstable $\mathrm{H}_{2} \mathrm{O}^{+}($I) cation final state. See the RPES photoemission spectra section in the main body of the text for further details.

considerable broadening of the RPES signals (with $\sim 0.2 \mathrm{eV}$, $\sim 1.0 \mathrm{eV}$, and $\sim 0.4 \mathrm{eV}$ broadenings expected near the $\mathrm{O} \mathrm{K}, \mathrm{Mn}$ $\mathrm{L}_{\mathrm{II}}$, and $\mathrm{Mn} \mathrm{L}_{\mathrm{III}}$ edges, respectively ${ }^{69}$ ). In an aqueous solution, the core-hole may be refilled in a number of different ways; for example, by electrons in higher-lying valence solute, and potentially solvent, ${ }^{70}$ molecular orbitals. Due to the elementally localised and fleeting nature of the core-hole, the refilling is expected to selectively occur via molecular orbitals with similar atomic parentages (Mn for Resonances I-IV and O for Resonances V-VI). However, if appreciable electron delocalisation for example, intra- or intermolecular charge transfer - occurs within the ultrashort core-hole lifetime, this refilling step may also be coupled to electron ejection from additional elements/ molecular orbitals. Equivalently considering such intra- or intermolecular processes in the energy domain, electron ejection from additional elements/molecular orbitals can occur if the photo-excited species are significantly involved in bonding interactions/are hybridised with the solute valence manifold.

Fig. $4 \mathrm{~B}_{1}$ illustrates an exemplary solute participator Auger decay process - where the solute core-hole is refilled by the electron that was promoted to the virtual valence orbital and a solute outer valence electron is simultaneously ejected. Notably, the process of refilling the core hole with the aforementioned solute outer valence electron and simultaneous ejection of the initially excited virtual-valence electron yields a similar electron kinetic energy and final state and is indistinguishable. Panel $\mathrm{C}_{1}$ shows the resulting final, singly oxidised solute $\left(\mathrm{MnO}_{4}{ }^{\circ}\right)$ state and highlights that such participator processes produce PES peaks with energy positions/nominal BEs similar to those generated through direct photoemission. Accordingly, such resonant enhancement features are identifiable due to their nominal BEs being near independent of the ionising photon energy. These participator Auger signals may also be significantly enhanced with respect to peaks in non-resonant/direct photoemission experiments due to both the increase in absorption cross-section at the excited resonances and potential constructive interference effects between the direct and participator Auger electron photoemission channels.

Fig. $4 \mathrm{~B}_{2}$ shows an exemplary alternative intramolecular decay process, a spectator resonant Auger channel - where the initially excited electron remains in a solute virtual-valence orbital, the core-hole is refilled by an outer-valence electron of the solute, and another solute outer valence electron is simultaneously ejected. Panel $\mathrm{C}_{2}$ shows the resulting electronically excited, singly oxidised $\left(\mathrm{MnO}_{4}{ }^{\circ}\right)$ final state. In this case, the peak position/ nominal $\mathrm{BE}$ of the emitted Auger electrons will vary with photon 
energy. However, their KEs will remain approximately constant at different resonances due to their dependence on the approximately constant molecular orbital energy gaps associated with the electrons involved in the de-excitation process. Panel $\mathrm{C}_{2}$ also implies that the energetics of the emitted spectator Auger electrons will be perturbed from the peak positions expected within a single-reference electronic structure framework. This is primarily due to the electron correlation differences between the electronically excited (valence) hole-pair spectator final states compared to the single-hole analogues produced through direct or participator resonant Auger ionisation.

With the solute embedded in an aqueous solvent, a number of non-local relaxation mechanisms may also occur, with the solvent molecules actively participating in the electronic deactivation processes. An example is shown in Fig. $4 \mathrm{~B}_{3}$, which shows a resonant ICD process involving a core-hole refill by a solute inner valence electron, with simultaneous ejection of an outer valence electron from a solvent molecule in the solute's first hydration shell. The final state of such an ICD process is shown in panel $\mathrm{C}_{3}$, which highlights production of an electronically excited solute anion $\left(\mathrm{MnO}_{4}{ }^{-}\right)$, with unchanged molecular charge, along with a highly unstable neighbouring $\mathrm{H}_{2} \mathrm{O}_{(1)}^{+}$cation. ${ }^{71}$ Such processes can be identified by their characteristic autoionization features ${ }^{72,73}$ which are expected to produce PES features with near photon energy independent electron KEs.

In all of the RPES processes described above, the relaxation mechanisms are driven by electron correlation effects in the initially-localised intermediate photo-excited states. In this regard, selective resonant enhancement of solute ionisation signals, the associated system energetics, and attribution of those signals to processes involving specific molecular orbitals, provides a framework to experimentally determine atomic parentages of RPES-active molecular orbitals (or more generally, electronic states). ${ }^{19,67,74}$ Conversely, a knowledge of the atomic parentage of specific molecular orbitals (states) can aid in the assignment of RPES features. Furthermore, should dynamic charge transfer processes occur within a core-hole lifetime - for example, from a metal centre to a ligand or from a ligand to a hydrating solvent molecule - signatures of such ultrafast events (or intramolecular or intermolecular hybridisation from an energy-domain perspective) are expected to be reflected in the RPES spectra. ${ }^{68}$

With the aim of confirming and disentangling overlapping solute BEs, assessing parentages of different solute valence molecular orbitals, and interrogating bonding interactions between the $\mathrm{MnO}_{4}{ }^{-}$solute and solvent molecules, the extended $\mathrm{NaMnO}_{4 \text { (aq.) }}$ solution spectra shown in Fig. 3 were analysed in detail. As a starting point, the baseline-corrected off-resonant spectra were subtracted from the corresponding baselinecorrected resonant spectra (see the Data treatment subsection for details). This isolated the enhancement signals at Resonances I, II, V, and VI (see Fig. 3). The Resonance III and IV data shown in ESI, $\dagger$ Fig. S7 was similarly treated. Fig. 5 shows the resonant-off-resonant difference spectra results for $\mathrm{Mn}\left(2 \mathrm{p}_{3 / 2}\right)$ Resonances I and II on a KE scale, with the peaks highlighting electronic relaxation processes involving molecular orbitals with significant Mn-character. Similar results are shown for the $\mathrm{Mn}$ $\left(2 \mathrm{p}_{1 / 2}\right)$ Resonances III and IV in Fig. S8 in the ESI. $\dagger$ Corresponding data for the $\mathrm{O}(1 \mathrm{~s})$ Resonances V and VI are shown in Fig. 6A, with the encompassed peaks highlighting resonant enhancement features involving molecular orbitals with significant O-ligand character. Cumulative Voigt or Gaussian profile fits were applied to the data shown in Fig. 5, 6 and Fig. S8 (ESI $\dagger$ ) to determine the energetics of the resonant enhancement peaks (see ESI, $\dagger$ Fig. S9). The results of these fits are summarized in Table $\mathrm{S} 4$ in the ESI. $\dagger$ Corresponding $\mathrm{KMnO}_{4(\mathrm{aq} .)}$ solution Resonance I, II, V, and VI results are shown in ESI, $\dagger$ Fig. S10, with associated fit results summarised in Table S5 (ESI $\dagger$ ). Based on these extracted RPES KEs/nominal BEs and the calibrated photon energy resonances, all RPES peaks have been assigned. Assignments were made by considering the experimental BEs determined from the direct PES experiments (see Table 1), excitation energy gaps from our $\mathrm{MnO}_{4}{ }^{-}$(aq.) $\mathrm{UV}$-visible (see Fig. S1 and ref. 56) and PEY-XAS spectra (Fig. 3 and Fig. S1, S7, ESI $\dagger$ ), and the $\mathrm{MnO}_{4}{ }^{-}$electronic structure calculation literature. ${ }^{40,54,56}$ Participator Auger peak feature assignments and averaged nominal BEs are reported in the bottom panel of Table 1, with the spectator Auger and ICD feature assignments and associated averaged electron KEs presented in Table 4. The rational for the RPES peak assignments is discussed in the following.

Mn 2p RPES assignments. We start by considering the $1 t_{2} /$ Mn 2p resonant PES data shown in Fig. 5. Beginning with the numbered participator Auger signals, the peak labelled 3-a in the Resonance II data dominates the signal enhancements, with an intensity 3.5 times that of the liquid water $1 b_{1}{ }^{-1}$ peak (see Fig. 3C). In this case, a single sharp peak with a $\mathrm{KE}$ of $633.0 \pm 0.1 \mathrm{eV}$ is extracted, corresponding to a participator $\mathrm{BE}$ of $12.66 \pm 0.04 \mathrm{eV}$. With reference to the direct PES BEs reported in Table 1 , this peak may be assigned to a $\mathrm{L}_{\mathrm{III}}\left(1 \mathrm{e} / 3 \mathrm{t}_{2}\right) 5 \mathrm{t}_{2}$ Auger process, involving the photoexcited $5 \mathrm{t}_{2}$ virtual valence and one

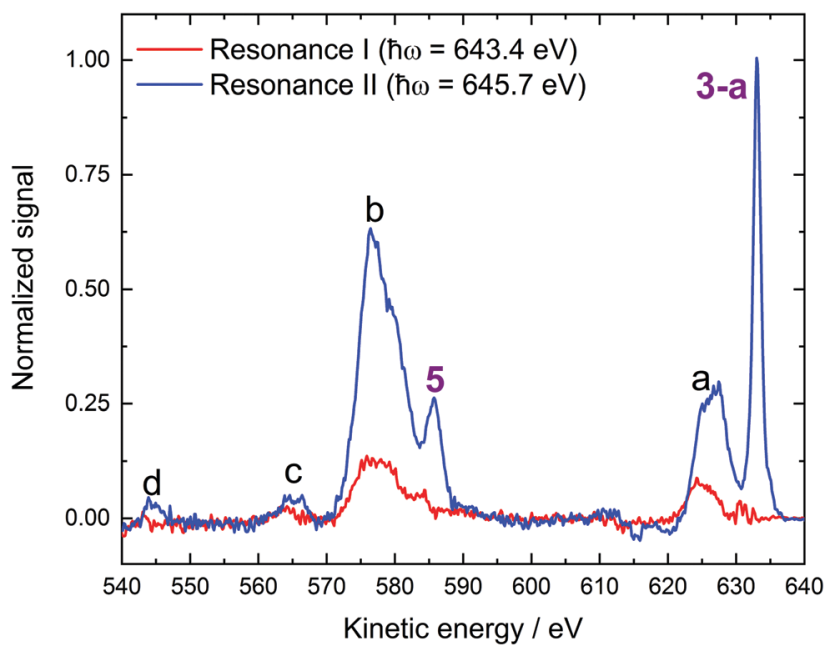

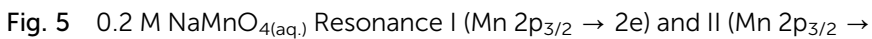
$5 t_{2}$ ) RPES difference spectra. Participator Auger signals are labelled with numbers while spectator auger signals are labelled with letters. The origins of the associated peaks are discussed in the main body of the text. 

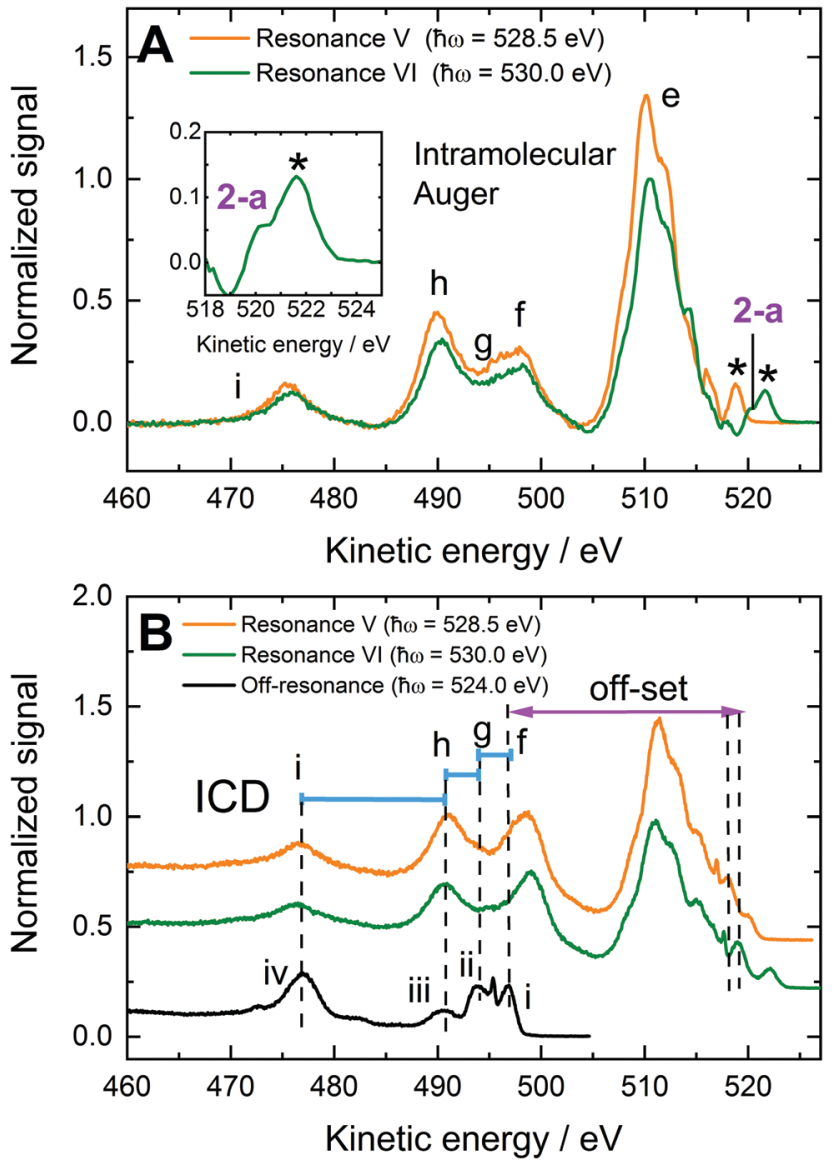

Fig. $6 \quad 0.2 \mathrm{M} \mathrm{NaMnO}_{4(\text { (aq.) }}$ solution Resonance $\mathrm{V}$ and $\mathrm{VI}$ RPES data. (A) Resonance $\mathrm{V}\left(\mathrm{O} 1 \mathrm{~s} \rightarrow 2 \mathrm{e}\right.$ ) and $\mathrm{VI}\left(\mathrm{O} 1 \mathrm{~s} \rightarrow 5 \mathrm{t}_{2}\right)$ RPES difference spectra. Participator Auger signals are labelled with numbers while spectator auger and potential ICD signals are labelled with letters. The asterisks highlight an experimental artefact signal associated with ionization of the $\mathrm{H}_{2} \mathrm{O}_{(1)} \mathrm{O}$ is molecular orbital with the residual, unfiltered second harmonic of the beam line. (B) As measured Resonance $V$ and $V I$ and spectrally offset off-resonant data highlighting the common energy gaps between peaks $f, g, h$, and $i$ and the water valence orbitals. Similar energetic separations of the resonantly enhanced RPES features and the water peaks in the offset non-resonant spectra imply that resonant ICD processes occur following ligand-core-tovirtual-valence excitations of $\mathrm{MnO}_{4}{ }^{-}$(aq.). The spectral offset applied to the off-resonant data corresponds to the sum of the resonant-non-resonant photon energy difference, the $5 a_{1}$ binding energy, and electron correlation effects. See the main body of the text for further details.

or both of the $1 \mathrm{e}$ and $3 \mathrm{t}_{2}$ outer valence molecular orbitals. We note that the general assignment of peak 3-a to a $1 \mathrm{e}^{-1}$ or $3 \mathrm{t}_{2}{ }^{-1}$ ionisation process (see the direct PES section and Table 1) is in agreement with the $\mathrm{MnO}_{4}{ }^{-}$valence molecular orbital descriptions of Jose et al. $^{40}$ and inferences regarding analogous solidphase data. ${ }^{67,75}$ The $12.66 \mathrm{eV}$ BE participator peak observation in the $1 \mathrm{t}_{2}\left(\mathrm{Mn} \mathrm{2} \mathrm{p}_{3 / 2}\right) \rightarrow 5 \mathrm{t}_{2}$ RPES spectra indicates that the 1e and/or $3 t_{2}$ outer valence (as well as the $5 t_{2}$ virtual valence) orbitals have significant $\mathrm{Mn}$ character. As shown in Table 1, a Gaussian FWHM value of $0.83 \pm 0.04 \mathrm{eV}$ was extracted for peak 3-a from a cumulative Voigt profile fit to the data. Comparison of this value to that observed from the direct PES results for peak 3 $(1.9 \pm 0.2 \mathrm{eV}$, see Table 1 , top) suggests that the direct PES feature
Table 4 Assignments of the RPES spectator Auger and solute-solvent ICD signals associated with the data shown in Fig. 3, 5 and 6. Many of the peaks were found to be made up of multiple components. The reported values correspond to the averaged KEs extracted at all resonances where such enhancement is observed. The KE associated with the dominant feature of each signal is highlighted in bold font. The ' $V$ ' peak label symbolises generic valence electron photoemission. The $(w)$ subscripts associated with some of the peak assignments highlight solute-water ICD core-hole relaxation mechanisms following excitation to Resonances $\mathrm{V}$ and VI

\begin{tabular}{lllc}
\hline Signal & Assignment 1 & Assignment 2 & $\mathrm{KE} / \mathrm{eV}$ \\
\hline $\mathrm{a}$ & $\mathrm{L}_{\mathrm{III}}-\mathrm{VV}$ & - & $\mathbf{6 2 7 . 1} \pm \mathbf{0 . 4}$ \\
$\mathrm{b}$ & $\mathrm{L}_{\mathrm{III}}-2 \mathrm{t}_{2}, \mathrm{~V}$ & - & $\mathbf{5 7 6} \pm \mathbf{1}$ \\
$\mathrm{c}$ & $\mathrm{L}_{\mathrm{III}}-2 \mathrm{t}_{2}, 5 \mathrm{a}_{1}$ & - & $\mathbf{5 6 5 . 7} \pm \mathbf{0 . 8}$ \\
$\mathrm{d}$ & $\mathrm{L}_{\mathrm{III}}-4 \mathrm{a}_{1}, \mathrm{~V}$ & - & $544.6 \pm 0.1$ \\
$\mathrm{e}$ & $\mathrm{K}-\mathrm{VV}$ & - & $516.3 \pm 0.1$ \\
& $\mathrm{~K}-\mathrm{VV}$ & - & $514.4 \pm 0.2$ \\
& $\mathrm{~K}-\mathrm{VV}$ & - & $512.2 \pm 0.1$ \\
& $\mathrm{~K}-\mathrm{VV}$ & - & $\mathbf{5 1 0 . 2} \pm \mathbf{0 . 2}$ \\
$\mathrm{f}$ & $\mathrm{K}-\mathrm{VV}$ & $\mathrm{K}-5 \mathrm{a}_{1}, \mathrm{~V}$ & $508.0 \pm 0.2$ \\
$\mathrm{~g}$ & $\mathrm{~K}-5 \mathrm{a}_{1}, 1 \mathrm{~b}_{1(\mathrm{w})}$ & $\mathrm{K}-5 \mathrm{a}_{1}, \mathrm{~V}$ & $\mathbf{4 9 8 . 3} \pm \mathbf{0 . 5}$ \\
$\mathrm{h}$ & $\mathrm{K}-5 \mathrm{a}_{1}, 3 \mathrm{a}_{1(\mathrm{w})}$ & - & $\mathbf{4 9 5} \pm \mathbf{1}$ \\
$\mathrm{i}$ & $\mathrm{K}-5 \mathrm{a}_{1}, 1 \mathrm{~b}_{2(\mathrm{w})}$ & - & $\mathbf{4 9 0 . 1} \pm \mathbf{0 . 2}$ \\
& $\mathrm{K}-5 \mathrm{a}_{1}, 2 \mathrm{a}_{1(\mathrm{w})}$ & - & $\mathbf{4 7 5 . 7} \pm \mathbf{0 . 2}$ \\
\hline
\end{tabular}

indeed has two underlying components and that a single ionisation channel component is selectively enhanced at Resonance II. We revisit the associated energetics and assignment of peak 3-a in the following section from an alternative perspective, based on our additional analysis of the $\mathrm{O} 1 \mathrm{~s}$ RPES spectra.

Our Voigt profile fits to the Resonance II data reveal a significant lifetime broadening contribution to the total width of peak 3-a ( $0.77 \pm 0.03 \mathrm{eV}$ FWHM Lorentzian component of a $1.32 \pm 0.01 \mathrm{eV}$ total FWHM peak width). This broadening likely reflects constructive interference between the direct $1 \mathrm{e}^{-1}$ or $3 \mathrm{t}_{2}{ }^{-1}$ ionization channels and the respective $\mathrm{L}_{\mathrm{III}}-1 \mathrm{e} 5 \mathrm{t}_{2}$ or $\mathrm{L}_{\mathrm{III}}-3 \mathrm{t}_{2} 5 \mathrm{t}_{2}$ participator Auger processes, which respectively result in the same final states. Indeed, average $\sim 0.4 \mathrm{eV}$ spectral broadenings are expected to be imparted onto (coherently) resonantly enhanced $\mathrm{L}_{\mathrm{III}}$-pre-edge peak widths, as channel interference exclusively occurs during the $\sim 1.8$ fs (average) $\mathrm{Mn} 2 \mathrm{p}_{3 / 2}$ core-hole lifetime. ${ }^{69}$ The greater lifetime broadening contribution observed

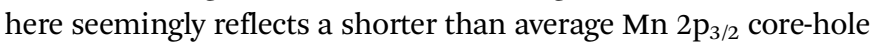
lifetime in the polyatomic, condensed-phase $\mathrm{MnO}_{4}{ }^{-}$(aq.) anion. Notably, similar results were obtained in the crystalline phase RPES studies reported by Reinert et al. ${ }^{67}$

A second Mn-associated participator Auger feature, peak 5, is observed in the Resonance I and II data shown in Fig. 5. These peaks occur at $583.9 \pm 0.1 \mathrm{eV}$ and $585.6 \pm 0.1 \mathrm{eV}$ KEs corresponding to respective $59.5 \pm 0.1 \mathrm{eV}$ and $60.1 \pm 0.1 \mathrm{eV}$ BEs $\left(\mathrm{BE}_{\text {average }}=59.8 \pm 0.4 \mathrm{eV}\right)$. Considering the $\mathrm{MnO}_{4}{ }^{-}$(aq.) $\mathrm{BES}$ determined in the direct PES experiments, peak 5 is readily assigned as a resonant enhancement of the $2 t_{2}$ (Mn 3p) inner valence ionisation feature. The resonant Auger processes responsible for peak 5 in the Resonance I and II difference spectra shown in Fig. 5 are therefore labelled $\mathrm{L}_{\mathrm{III}}-2 \mathrm{t}_{2} 2 \mathrm{e}$ and $\mathrm{L}_{\mathrm{III}}-2 \mathrm{t}_{2} 5 \mathrm{t}_{2}$, respectively.

We now consider the spectator Auger decay channel contributions to Fig. 5 (and the equivalent $\mathrm{KMnO}_{4}{ }^{-}$(aq.) data shown 
in Fig. S10A and B, ESI $\dagger$ ). Starting with feature a, a spectral profile that appears to be made up of two or more peaks is observed to contribute to both the Resonance I and Resonance II spectra. The KE of the main feature is reported in Table 4. Similar features occur following excitation to Resonances III and IV, as shown in Fig. S8 in the ESI. $\dagger$ Feature a is notably narrower in the Resonance I and II cases ( $2 \mathrm{p}_{3 / 2}$ core-hole) with respect to the Resonance III and IV cases ( $2 \mathrm{p}_{1 / 2}$ core-hole), as expected based on the shorter $\mathrm{L}_{\mathrm{II}^{-}}$versus $\mathrm{L}_{\mathrm{III}}$-edge core-hole lifetimes of the latter $\left(\sim 1.8 \mathrm{fs}\right.$ versus $\sim 0.7 \mathrm{fs}$, on average, respectively $\left.{ }^{69}\right)$. Furthermore, additional lower KE relaxation channels seemingly become accessible following higher-energy excitation to Resonances III and IV. Upon consultation of Table 1, feature a is generally attributed to L-VV spectator Auger processes, involving different pairs of $\mathrm{Mn}-\mathrm{O}$ outer valence $(\mathrm{V})$ bonding permanganate orbitals $\left(6 \mathrm{a}_{1}, 1 \mathrm{e}, 3 \mathrm{t}_{2}\right)$. The broadening and spectral extension of feature a in the Resonance III and IV cases is then attributed to the onset of $\mathrm{L}_{\mathrm{II}}-\mathrm{L}_{\mathrm{III}} \mathrm{V}$ Coster-Kronig channels, ${ }^{76}$ as described in the ESI. $\dagger$

Similarly to feature a, feature b appears to encompass two or more RPES peaks. Again, the KE of the main feature is reported in Table 4 and similar, but broadened, features are observed in the Resonance III and IV data shown in ESI, $\dagger$ Fig. S8. We generally attribute the peak b signal components to solute spectator Auger processes involving the $2 \mathrm{t}_{2}$ (Mn 3p) inner valence and solute $\mathrm{Mn}-\mathrm{O}$ bonding outer valence electrons $\left(6 \mathrm{a}_{1}, 1 \mathrm{e}, 3 \mathrm{t}_{2}\right)$, i.e. $\mathrm{L}_{\mathrm{III}}-2 \mathrm{t}_{2} \mathrm{~V}$. The appearance of such peaks are indicative of outer-inner valence electronic coupling, i.e. $\mathrm{Mn} 3 \mathrm{p}$ hybridisation. Additionally, noting that the $6 \mathrm{a}_{1}$ orbital is predicted to have significant Mn 3p character - unlike the $1 \mathrm{e}$ and $3 \mathrm{t}_{2}$ orbitals - peak $\mathrm{b}$ may be tentatively primarily attributed to a $\mathrm{L}_{\mathrm{III}}-2 \mathrm{t}_{2} 6 \mathrm{a}_{1}$ spectator Auger process. ${ }^{40}$ As with peak a, $\mathrm{L}_{\mathrm{II}}-\mathrm{L}_{\mathrm{III}} 2 \mathrm{t}_{2}$ Coster-Kronig transitions lead to a relative broadening and spectral extension of feature $b$ in the Resonance III and IV excitation cases (see the ESI $\dagger$ for details).

A relatively weak additional spectator Auger signal - labelled peak c - occurs at an average $\mathrm{KE}$ value of $565.7 \pm 0.8 \mathrm{eV}$ and is present in both the Resonance I and II spectra. We tentatively assign these peaks to a $\mathrm{L}_{\mathrm{III}}-2 \mathrm{t}_{2} 5 \mathrm{a}_{1}$ spectator Auger process. This feature in particular suggests that the inner valence $2 t_{2}$ (Mn 3p) and $5 \mathrm{a}_{1}$ (O 2s) and resonantly populated $2 \mathrm{e}$ and $5 \mathrm{t}_{2}$ virtual valence orbitals are coupled and that the $\mathrm{Mn}$ and O inner valence atomic orbitals are hybridised within the valence manifold. The corresponding signals in the Resonance III and IV data are thought to be buried under the aforementioned Coster-Kronig features.

Finally, additional weak resonantly-enhanced signals are observed in the Resonance I and II spectra at KEs close to $544.2 \pm 0.1 \mathrm{eV}$. We label these features $\mathrm{d}$ and assign them to a $\mathrm{L}_{\mathrm{III}}-4 \mathrm{a}_{1} \mathrm{~V}$ spectator Auger emission process. Considering the $\mathrm{MnO}_{4}{ }^{-}$(aq.) $4 \mathrm{a}_{1}$ (Mn 3s) BE from Table 1 and neglecting electron correlation effects, we estimate a BE of $\sim 12.5 \mathrm{eV}$ for the orbital involved in the $\mathrm{L}_{\mathrm{III}}-4 \mathrm{a}_{1} \mathrm{~V}$ process. This $\mathrm{BE}$ value is consistent with those extracted from signal 3 and RPES signal 3-a, suggesting a $\mathrm{L}_{\mathrm{III}}-4 \mathrm{a}_{1} 1 \mathrm{e}$ and/or $\mathrm{L}_{\mathrm{III}}-4 \mathrm{a}_{1} 3 \mathrm{t}_{2}$ process occurs. Such processes highlight the Mn-based character of the $1 \mathrm{e}$ and/or $3 \mathrm{t}_{2}$ orbitals, associated electronic coupling to the $4 \mathrm{a}_{1}$ orbitals, and Mn $3 \mathrm{~s}$ inner-outer valence hybridisation.

O 1s RPES assignments - role of intramolecular Auger processes. The Auger signals extracted from the Resonance V and VI RPES spectra shown in Fig. 6A result from photoexcitation from the O-centred core ligand orbitals into unoccupied metal-ligand antibonding orbitals. In general, the reduced lifetime broadening of the associated intermediate states (with respect to those produced following excitation at Resonances I-IV), permit a more ready resolution of individual solute Auger relaxation channels.

We first consider the solute participator Auger decay channels. Given the non-bonding, predominant $\mathrm{O} 2 \mathrm{p}$ character of the $1 \mathrm{t}_{1}$ and $4 \mathrm{t}_{2}$ orbitals, $^{40,56}$ associated participator Auger signals are expected to emerge in the 9-11 eV BE range (see Table 1). However, the analysis of this spectral region is complicated by its overlap with beamline second harmonic artefact signals (peaks marked by '*' in Fig. 3E, F and 6A). Particularly, the Resonance V participator Auger decay channel associated with the $1 t_{1}$ molecular orbital would be superimposed on this artefact. Hence, such a feature cannot be resolved using the difference spectrum shown in Fig. 6A alone. However, the $\mathrm{K}-2 \mathrm{e} 1 \mathrm{t}_{1}$ participator peak is discernible from the photon energy dependence of the RPES map shown in Fig. 3D. This map specifically highlights an enhanced BE feature at $9.2 \pm 0.1 \mathrm{eV}$ over a $\sim 1 \mathrm{eV}$ photon energy region in the vicinity of Resonance $\mathrm{V}$, in agreement with the $\mathrm{BE}$ extracted from the direct PES spectra.

More generally, participator Auger decay signals are identifiable from the high signal-to-noise ratio, background subtracted spectra, like those presented in Fig. 6A. In the Resonance VI data, the lower KE shoulder to the second harmonic artefact signal (marked '*' in Fig. 6A) is labelled peak 2-a and resolved at a KE of $520.1 \pm 0.1 \mathrm{eV}$, corresponding to a BE of $9.85 \pm 0.08 \mathrm{eV}$. Consulting Table 1 and noting the aforementioned predominant oxygen electronic character of the $4 \mathrm{t}_{2}$ molecular orbital, we ascribe this feature to a $\mathrm{K}-4 \mathrm{t}_{2} 5 \mathrm{t}_{2}$ process. The $9.85 \pm 0.08 \mathrm{eV} \mathrm{BE}$ is consequently appended to a $4 \mathrm{t}_{2}{ }^{-1}$ ionisation channel, with the associated BE included in the bottom panel of Table 1 with a peak 2-a label. In this particular case, we note that the reported $\mathrm{BE}$ may be somewhat biased by over-subtraction of the off-resonant background data. Although our results are consistent across multiple data sets which display varying degrees of over-subtraction, the participator $\mathrm{BE}$ value reported must, therefore, be considered a lower limit of the true value. We further note that any similar participator Auger signals in the Resonance $\mathrm{V}$ data would be buried under the second harmonic artefact signal.

The $4 \mathrm{t}_{2}{ }^{-1} \mathrm{BE}$ peak $(9.85 \pm 0.08 \mathrm{eV}, 2$-a) discerned in the O-sensitive Resonance VI RPES data sets displays a relatively narrow average width $(0.7 \pm 0.2 \mathrm{eV})$ in comparison to the peak 2 feature extracted from the direct PES results $(1.2 \pm 0.1 \mathrm{eV}$ at FWHM). Therefore, with the consistency of the RPES feature width across multiple data sets in mind, we infer that peak 2 in the direct PES experiments indeed consists of two overlapping components. The broader associated spectral profile would 
then correspond to the weighted average of the $4 \mathrm{t}_{2}{ }^{-1}$ and $6 \mathrm{a}_{1}{ }^{-1}$ features, resulting in the extracted peak $2 \mathrm{BE}$ of $10.67 \pm 0.04 \mathrm{eV}$. We note that this inference is further supported by the solid-phase ultraviolet PES $\mathrm{KMnO}_{4}$ experiments reported by Reinert et al., ${ }^{37}$ which resolved the solid-state analogues of the peak 2 components using high-energy-resolution, near-threshold ionisation.

Additional participator Auger features potentially occur in the 515-520 eV KE region, although they cannot be unequivocally assigned. A particularly weak feature is observed close to a $518 \mathrm{eV} \mathrm{KE}$ in the Resonance VI data, which may be linked to a $\mathrm{L}_{\mathrm{III}}-5 \mathrm{t}_{2} 6 \mathrm{a}_{1}$ participator channel and relate to direct PES peak 2 and RPES peak 2-a. However, the KE and width of this feature cannot be reliably extracted due to the low signal intensity and the over-subtraction of the off-resonant spectrum in this region. Another, more intense, potential participator feature occurs at a $\mathrm{KE}$ of $516.3 \pm 0.1 \mathrm{eV}$ in the Resonance $\mathrm{V}$ data, corresponding to a calibrated BE of $12.18 \pm 0.05 \mathrm{eV}$. Considering our assignment of peak 3-a to a selective enhancement of either the $1 \mathrm{e}^{-1}$ or $3 \mathrm{t}_{2}{ }^{-1}$ ionization channels, the $516.3 \pm 0.1 \mathrm{eV}$ peak could potentially be assigned as a participator Auger peak and to a selective $\mathrm{K}-1 \mathrm{e} 5 \mathrm{t}_{2}$ ionisation process, with peak 3-a correspondingly being attributed to a selective $\mathrm{L}_{\mathrm{III}}-3 \mathrm{t}_{2} 5 \mathrm{t}_{2}$ participator Auger process. Hence, a 1e BE value of $12.18 \pm 0.05 \mathrm{eV}$ and a $3 t_{2}$ BE value of $12.66 \pm 0.04 \mathrm{eV}$ (peak 3-a) could be tentatively inferred. Following such an inference, peak 3 in the direct PES experiments (with an extracted BE of 12.84 \pm $0.09 \mathrm{eV}$ ) would then be associated with a weighted average of the two RPES participator peaks (with peak 3-a making the dominant contribution to the cumulative peak detected in direct PES). As appealing as such an assignment may be, we caution, however, that the $\sim 518 \mathrm{eV}$ and $516.3 \pm 0.1 \mathrm{eV}$ potential participator peaks mentioned in this paragraph may be additionally and (perhaps more convincingly) alternatively attributed to spectator Auger processes, as discussed below. As such, the individual $1 \mathrm{e}$ and $3 \mathrm{t}_{2}$ BEs cannot be unambiguously determined, neither in our direct PES nor RPES experiments.

Focusing on the 9.0-10.5 eV participator BE region, a notable absence of any $1 \mathrm{t}_{1}$ or $4 \mathrm{t}_{2}$ orbital participator signals in the Mn-sensitive spectra (which are unperturbed by artefact signals) is highlighted. This confirms the predominant O-character of these molecular orbitals. Considering the higher $\mathrm{BE} 6 \mathrm{a}_{1}, 1 \mathrm{e}$, and $3 \mathrm{t}_{2}$ participator signals in the $\mathrm{O} \mathrm{K}$-pre-edge data, only features potentially associated with the $1 \mathrm{e}$ molecular orbital could be identified. Additional signatures of the $6 \mathrm{a}_{1}$, $1 \mathrm{e}$, and $3 \mathrm{t}_{2}$ ionisation channels are either too weak, or are obscured by the beamline second harmonic artefact signal or K-VV spectator Auger (and potentially non-local intermolecular) decay signatures. Therefore, further insights into the $\mathrm{O}$ character and BEs of the $6 \mathrm{a}_{1}, 1 \mathrm{e}$, and $3 \mathrm{t}_{2}$ molecular orbitals can only be garnered through analysis of the spectator Auger signals, which we discuss in the following.

Considering the spectator Auger signals in Fig. 3E, F and 6A (and the $\mathrm{KMnO}_{4 \text { (aq.) }}$ data shown in Fig. S6 and S10, ESI $\dagger$ ), a structured feature spanning KEs of $505-519 \mathrm{eV}$ is found to dominate the Resonance V and VI spectra. This structure, labelled peak e, constitutes a manifold/convolution of four or more components with $\sim 2.1 \mathrm{eV}$ sub-structure separations. We note that a very similar feature was observed in the solid-state $\mathrm{KMnO}_{4}$ RPES data reported by Reinert et al., ${ }^{67}$ demonstrating that these peaks are associated with the $\mathrm{MnO}_{4}{ }^{-}$anion. The energy range spanned by feature e indicates its components correspond to K-VV spectator Auger processes. Possible processes associated with the $514.4 \pm 0.2 \mathrm{eV}, 512.2 \pm 0.1 \mathrm{eV}$, $510.2 \pm 0.2 \mathrm{eV}$, and $508.0 \pm 0.2 \mathrm{eV} \mathrm{KE}$ peaks include the $\mathrm{K}-1 \mathrm{t}_{1} 3 \mathrm{t}_{2}, \mathrm{~K}-1 \mathrm{e} 1 \mathrm{e}, \mathrm{K}-6 \mathrm{a}_{1} 3 \mathrm{t}_{2}$, and $\mathrm{K}-3 \mathrm{t}_{2} 3 \mathrm{t}_{2}$ spectator Auger decay channels. Alternative and related assignments for the $516.3 \pm$ $0.1 \mathrm{eV}$ feature in the Resonance $\mathrm{V}$ data and the $\sim 518 \mathrm{eV} \mathrm{KE}$ peak in the Resonance VI data - discussed in the context of potential participator Auger channels above - would then be respective $K-4 t_{2} 4 t_{2}$ and $K-1 t_{1} 1 t_{1}$ processes. Indeed, when the common $\sim 2.1 \mathrm{eV}$ sub-peak separations in the feature $\mathrm{e}$ progression are considered, we favour the assignment of the $516.3 \pm 0.1 \mathrm{eV}$ and $\sim 518 \mathrm{eV}$ features to the aforementioned resonant spectator Auger processes (although the abovediscussed participator channels may additionally underlie these peaks). We note that the resolution of up to six distinct spectral features spanning the peak e energy range and their assignment to K-VV spectator Auger processes suggests the entire valence band of $\mathrm{MnO}_{4}{ }^{-}$(aq.) is characterised by significant $\mathrm{O}$ character.

The signals spanning the KE range from 470-505 eV appear to be made up of three unresolved components and a lower $\mathrm{KE}$ feature that we label features $\mathrm{f}, \mathrm{g}, \mathrm{h}$ and feature $\mathrm{i}$, respectively. These features have remarkably similar profiles in the Resonance $\mathrm{V}$ and VI RPES spectra recorded from all $\mathrm{MnO}^{-}{ }^{-}$(aq.) solutions. They are also significantly broadened with respect to the subcomponents of signal e; from which multiple features separated by just $\sim 2 \mathrm{eV}$ could be isolated. The electron KEs associated with signals f, $\mathrm{g}, \mathrm{h}$ and $\mathrm{i}$ were determined to be $498.3 \pm 0.5 \mathrm{eV}$, $495 \pm 1 \mathrm{eV}, 490.1 \pm 0.2 \mathrm{eV}$, and $475.7 \pm 0.2 \mathrm{eV}$, respectively, via a multi peak fit analysis (see ESI, $\dagger$ Fig. S9, S10 and Tables S4, S5). These energetics indicate involvement of the $\mathrm{MnO}_{4}{ }^{-}$(aq.) nominally non-bonding inner valence $5 \mathrm{a}_{1}$ (O 2s) electrons, with the features potentially being assignable to $\mathrm{K}-5 \mathrm{a}_{1} \mathrm{~V}$ spectator Auger processes. The appearance of such peaks are again indicative of outer-inner valence orbital electronic coupling in $\mathrm{MnO}_{4}{ }^{-}$(aq.), i.e. in this case, $\mathrm{O} 2 \mathrm{~s}$ valence hybridisation. Considering the $5 \mathrm{a}_{1} \mathrm{BE}$ from the direct PES experiments (see Table 1), the following peak $\mathrm{f}, \mathrm{g}, \mathrm{h}$ and $\mathrm{i}$ BEs are respectively estimated (neglecting electron correlation): $10.9 \pm 0.5 \mathrm{eV}, 14 \pm 1 \mathrm{eV}, 19.1 \pm 0.2 \mathrm{eV}$, and $33.5 \pm$ $0.2 \mathrm{eV}$. Possible intramolecular solute spectator Auger assignments for peaks $f$ and $g$ would be K-5a $\mathrm{a}_{1}\left(4 \mathrm{t}_{2} / 6 \mathrm{a}_{1}\right)$ and $\mathrm{K}-5 \mathrm{a}_{1}\left(1 \mathrm{e} / 3 \mathrm{t}_{2}\right)$, respectively. However, peak $\mathrm{h}$ is more challenging to assign, with no solute BEs lying within $\pm 6 \mathrm{eV}$ of the expected $\sim 19 \mathrm{eV}$ nominal $\mathrm{BE}$ of a $5 \mathrm{a}_{1}$ spectator Auger partner electron. Peak i also has no obvious spectator Auger signal assignment, with the closest match to its energetics being a $\mathrm{K}-5 \mathrm{a}_{1} 5 \mathrm{a}_{1}$ process that would be shifted by more than $6 \mathrm{eV}$ to higher $\mathrm{KE}$ in comparison to singlereference electronic structure expectations. With these assignment challenges in mind, we are driven to consider alternative, non-local core-hole relaxation mechanisms for the assignment of peaks $\mathrm{h}$ and $\mathrm{i}$, and potentially $\mathrm{f}$ and $\mathrm{g}$. 
O 1 S RPES assignments - role of non-local autoionization processes. A clue regarding the origin of features $f, g, h$, and $i$ is provided by the energetic separation of the neighbouring peaks: $3 \pm 1 \mathrm{eV}, 5 \pm 1 \mathrm{eV}$, and $14.4 \pm 0.3 \mathrm{eV}$. Within our error bounds, these peak separations closely match the energy gaps between the valence BEs of liquid water (when average, 13.74 \pm $0.08 \mathrm{eV}, 3 \mathrm{a}_{1(\mathrm{l})}$ BEs are considered, see Table 2). Furthermore, the absolute KE positions of these photoelectron features are in agreement with those expected for $\mathrm{K}-5 \mathrm{a}_{1} \mathrm{~V}_{(\mathrm{w})}$ relaxation processes, where the $(\mathrm{w})$ sub-scripts appended to the valence molecular orbitals refer to those of water molecules in the first solute hydration shell. Omitting electron correlation effects - particularly associated with the electrons promoted to the solute virtual valence $\left(2 \mathrm{e}, 5 \mathrm{t}_{2}\right)$ orbitals through photoexcitation at Resonances V and VI - a $5 \mathrm{a}_{1}(\mathrm{O} 2 \mathrm{~s}) \rightarrow 3 \mathrm{a}_{1}(\mathrm{O} 1 \mathrm{~s})$ core-hole refill process results in a remaining system excitation energy of up to $536.0 \pm 0.1 \mathrm{eV}\left(\mathrm{BE}_{3 \mathrm{a} 1 / \mathrm{O} 1 \mathrm{~s}}\right)-26.82 \pm 0.04 \mathrm{eV}$ $\left(\mathrm{BE}_{5 \mathrm{a} 1 / \mathrm{O} 2 \mathrm{~s}}\right)=509.2 \pm 0.1 \mathrm{eV}$. Considering an associated energy transfer from the solute to solvent molecules and ejection of water $1 b_{1}, 3 a_{1}, 1 b_{2}$, and $2 a_{1}$ valence electrons, respective electron KEs of $497.9 \pm 0.1 \mathrm{eV}, 495.5 \pm 0.1 \mathrm{eV}, 491.8 \pm 0.1 \mathrm{eV}$, and $478.2 \pm 0.1 \mathrm{eV}$ are expected. The agreement of these predicted KEs with the measured $\mathrm{f}, \mathrm{g}$, h, and i peak positions (see the previous sub-section and Tables 2 and 4 ) is deemed to be fully consistent with the expected shielding/electron correlation effects of the photoexcited solute virtual valence electrons and our experimental uncertainties. Hence, the predominant electronic relaxation pathways associated with features $\mathrm{f}, \mathrm{g}$, h, and $\mathrm{i}$ are denoted as $\mathrm{K}-5 \mathrm{a}_{1} 1 \mathrm{~b}_{1(\mathrm{w})}, \mathrm{K}-5 \mathrm{a}_{1} 3 \mathrm{a}_{1(\mathrm{w})}$, $\mathrm{K}-5 \mathrm{a}_{1} 1 \mathrm{~b}_{1(\mathrm{w})}$, and $5 \mathrm{a}_{1}, 1 \mathrm{~b}_{1(\mathrm{w})}$ ICD processes, respectively. We support and schematically summarise these assignments in Fig. 6B.

In Fig. 6B, we KE offset the off-resonant spectrum recorded at $524.0 \pm 0.1 \mathrm{eV}$ by the difference in the photon energies used to record the off-resonant and Resonance $\mathrm{V}$ and VI data $\left(+4.5 \pm 0.1 \mathrm{eV}\right.$ and $+6.0 \pm 0.1 \mathrm{eV}$, respectively) and the $5 \mathrm{a}_{1} \mathrm{BE}$ $(-26.82 \pm 0.04 \mathrm{eV})$. This leads to overall off-resonant KE shifts of $-22.3 \pm 0.1 \mathrm{eV}$ and $-20.8 \pm 0.1 \mathrm{eV}$ with respect to the Resonance $\mathrm{V}$ and VI data, respectively. As a result, we see an excellent overlap keeping in mind the additional differential peak shifting associated with electron correlation effects - of the water valence feature peak centres with the spectrally broadened $\mathrm{f}, \mathrm{g}$, h, and i features, which we attribute to resonant ICD.

We note that such ICD-induced mirroring of the liquid water photoelectron spectrum in RPES data has previously been observed following core-to-virtual valence resonant excitation of $\mathrm{OH}^{-}$(aq.) ${ }^{33}$ In that case, participator-like ICD processes involving core-hole refill by the initially photoexcited electrons and simultaneous ejection of valence electrons from the solvent - were observed; as opposed to spectator-like $\left(\mathrm{K}-5 \mathrm{a}_{1} \mathrm{~V}_{(\mathrm{w})}\right)$ ICD processes ascribed to peaks $\mathrm{f}, \mathrm{g}, \mathrm{h}$, and i in Fig. 6. Consequently, the question of whether such participator-ICD processes also contribute to the $\mathrm{MnO}_{4}{ }^{-}$(aq.) $\mathrm{O} \mathrm{K}$ pre-edge data shown in Fig. 6 arises. Using similar arguments to those presented above, we can estimate the KE positions for such participator-like ICD electrons in Fig. 6 . For water $1 b_{1}, 3 a_{1}, 1 b_{2}$, and $2 \mathrm{a}_{1}$ electron ejection, respective KEs of $517.2 \pm 0.1 \mathrm{eV}$, $514.8 \pm 0.1 \mathrm{eV}, 511.1 \pm 0.1 \mathrm{eV}$, and $497.5 \pm 0.1 \mathrm{eV}$ and $518.7 \pm 0.1 \mathrm{eV}, 516.3 \pm 0.1 \mathrm{eV}, 512.6 \pm 0.1 \mathrm{eV}$, and $499.0 \pm$ $0.1 \mathrm{eV}$ are expected at Resonances $\mathrm{V}$ and VI, respectively. Indeed, these KEs span the range of feature e in Fig. 6. However, the sharpness of the ionisation features in these spectral regions and the occurrence of a similar spectral profile in crystalline $\mathrm{KMnO}_{4} \mathrm{RPES}$ data, ${ }^{67}$ imply that intramolecular spectator Auger decay processes dominate in the feature e region.

We finally emphasise that the resonant ICD features - being signatures of electronic coupling between the $\mathrm{MnO}_{4}{ }^{-}$(aq.) solute ligands and directly hydrating solvent molecules - were uniquely accessible in our RPES experiments, and only following detailed and careful analysis of the participator and spectator Auger channels. The relative intensity of resonant enhancement features $\mathrm{f}, \mathrm{g}, \mathrm{h}$, and $\mathrm{i}$, and particularly the occurrence of peaks $h$ and $i$ in what would otherwise be a sparsely populated region of the RPES spectrum, highlights the great potential of liquid-phase RPES to interrogate intermolecular bonding phenomena in the aqueous-phase, even at relatively low solute concentrations $(0.2 \mathrm{M}$ versus a $\sim 56 \mathrm{M}$ water solvent concentration).

\section{Absolute energetics and thermodynamics of $\mathrm{MnO}_{4}{ }^{-}$(aq.) solutions}

Having extensively surveyed the electronic structure of $\mathrm{MnO}_{4}{ }^{-}$(aq.) in the previous sections, we utilise a selection of our results to extract aqueous-phase energetic parameters that underpin redox and more general chemical phenomena involving $\mathrm{MnO}_{4}{ }^{-}$(aq.) Of particular interest are the aqueous-phase adiabatic ionisation energies, $\operatorname{AIE}_{(\text {aq.) }}$, characteristic minimum energy thresholds for liberating electrons to vacuum. With chemical reactions - including redox processes - primarily governed by the absolute energetics of the most readily liberated electrons, we focus our initial analysis on the lowest energy vertical ionising transition of $\mathrm{MnO}_{4}{ }^{-}$(aq.) $\left(1 \mathrm{t}_{1}{ }^{-1}\right)$, the associated production of the $(1)^{2} \mathrm{~T}_{1}$ state of $\mathrm{MnO}_{4}{ }^{\bullet}$ radical, and the $\mathrm{MnO}_{4}{ }^{-} / \mathrm{MnO}_{4}{ }^{\bullet}+\mathrm{e}^{-}$redox half reaction. We begin by defining the free energetics of the associated chemical cycle.

Fig. 7A highlights the free energetic relationships between the VIE and AIE of $\mathrm{MnO}_{4}{ }^{-}$(aq.), the VEA of $\mathrm{MnO}_{4}{ }^{\circ}$ (aq.), and the associated reorganisation free energies of oxidation $\left(\lambda_{\text {ox }}\right)$ and reduction $\left(\lambda_{\text {red }}\right)$. The diagram depicts the following relation that can be utilised to extract the lowest AIE of $\mathrm{MnO}_{4}{ }^{-}$(aq.), equivalent to its Gibbs free energy of oxidation, $\Delta G_{\text {ox }}$ :

$$
\mathrm{AIE}=\mathrm{VIE}-\lambda_{\mathrm{ox}}=\Delta G_{\mathrm{ox}}
$$

It has previously been demonstrated that, within the linear response approximation, liquid-phase photoelectron feature widths can be related to $\lambda_{\mathrm{ox}}$ using the equation below: ${ }^{12-14,77,78}$

$$
\lambda_{\mathrm{ox}}=\frac{\left(\mathrm{FWHM}_{\mathrm{IB}} / 2 \sqrt{2 \ln 2}\right)^{2}}{2 k_{\mathrm{B}} T}
$$




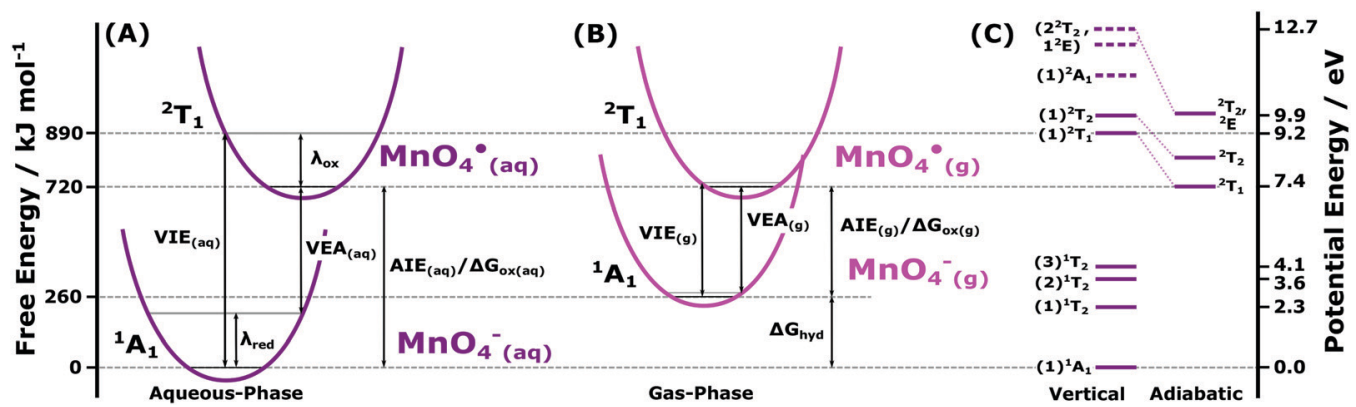

Fig. 7 (A) Schematic quadratic potential energy curves of the $\mathrm{MnO}_{4}^{-}$(aq.) anion and $\mathrm{MnO}_{4}{ }^{{ }^{\circ}}$ (aq). radical, highlighting the parameters extracted from the lowest energy $\mathrm{MnO}_{4}{ }^{-}$(aq.) ionising transition. (B) Corresponding schematic of the $\mathrm{MnO}_{4}{ }^{-}$(g) anion and $\mathrm{MnO}_{4}{ }^{\bullet}$ (g) radical potential energy curves, produced according to ref. 34 and energetically aligned so that the ground rovibronic state of the $\mathrm{MnO}_{4}$. radical occurs at the same energy in the aqueous- and gas-phase. the associated alignment energy corresponds to the vacuum level or $+7.4 \mathrm{eV}^{-}$on the potential energy scale (relative to the $\mathrm{MnO}_{4}{ }^{-}$(aq.) anion ground state). (C) Electronic energy level structure of $\mathrm{MnO}_{4}{ }^{-}$(aq.) and $\mathrm{MnO}_{4}{ }^{{ }^{(} \text {(aq.) }}$ extracted from the spectroscopic results reported herein and potential energy referenced to the $\mathrm{MnO}_{4}{ }^{-}$(aq.) electronic ground state. On the left of diagram (C), vertical excitation energies and associated state energy levels of the optically active anion excited states of $\mathrm{MnO}_{4}{ }^{-}$(aq.) (see Fig. S1A in the ESI $\dagger$ ) and valence electronically excited states of the $\mathrm{MnO}_{4}{ }^{\circ}$ (aq.) radical are shown. The solid lines are associated with the precisely determined $\mathrm{MnO}_{4}{ }^{-}$(aq.) and $\mathrm{MnO}_{4}{ }^{{ }^{*}}$ (aq.) state energies, with dashed lines denoting the equivocally inferred $(1)^{2} \mathrm{~A}_{1}\left(6 \mathrm{a}_{1}{ }^{-1}\right),(1)^{2} \mathrm{E}\left(1 \mathrm{e}^{-1}\right)$, and $(2)^{2} \mathrm{~T}_{2}\left(3 \mathrm{t}_{2}{ }^{-1}\right) \mathrm{MnO}_{4}{ }^{-}$(aq.) state energetics at the equilibrium geometry of the $\mathrm{MnO}_{4}{ }^{-}$(aq.) ground state. For the two lowest $\mathrm{VIES}$ corresponding adiabatic $\mathrm{MnO}_{4}{ }^{\circ}$ (aq.) radical state energy levels, as extracted using the direct PES $\left({ }^{2 \mathrm{~T}}{ }_{1}\right)$ and $\left({ }^{2 \mathrm{~T}}{ }_{2}\right) \mathrm{RPES}$ peak BEs/widths and linear response theory, are shown to the right. VIE: vertical ionisation energy, VEA: vertical electron affinity, $\lambda_{\text {ox/red: }}$ total reorganisation energy of the oxidised/reduced state, AIE: adiabatic ionisation energy, $\Delta G_{\text {ox }}$ : Gibbs free energy of oxidation, $\Delta G_{\text {hyd: }}$ Gibbs free energy of hydration.

where FWHM $_{\mathrm{IB}}$ characterizes the Gaussian width of the inhomogeneous broadening contribution to the photoelectron peak, $k_{\mathrm{B}}$ is the Boltzmann constant, and $T$ is the solution temperature. Therefore, using the lowest energy ionising transition feature of $\mathrm{MnO}_{4}{ }^{-}$(aq.) reported here (see peak 1 in Fig. 2 and Table 1) and expressions (2) and (1), it is possible to estimate both $\lambda_{\text {ox }}$ and the $\mathrm{AIE}$ of $\mathrm{MnO}_{4}{ }^{-}$(aq.)

Notably, approximating the $\mathrm{FWHM}_{\mathrm{IB}}$ term in eqn (2) with a photoelectron feature width (for example, the average values reported in Table 1) will over and under estimate the values of $\lambda_{\text {ox }}$ and AIE, respectively. The overall ionisation peak width is only partially determined by the inhomogeneous broadening term, being further widened by experimental resolution limitations and potential lifetime broadening effects. Only by carefully accounting for the latter broadening mechanisms, can we expect to extract accurate $\mathrm{FWHM}_{\mathrm{IB}}$ terms and, hence, values of $\lambda_{\text {ox }}$ and $\operatorname{AIE}_{(\text {aq. })}$.

The Gaussian fits to our direct PES spectra recorded at sample temperatures of $277-278 \mathrm{~K}$ yield an average $0.8 \pm$ $0.1 \mathrm{eV} 1 \mathrm{t}_{1}{ }^{-1}$ (peak 1) feature width that we term $\mathrm{FWHM}_{\mathrm{G}}$. As previously mentioned, our direct PES data analysis confirmed that lifetime broadening effects are negligible in the associated valence spectral region. The solution phase $\mathrm{FWHM}_{\mathrm{IB}}$ component for the $1 \mathrm{t}_{1}{ }^{-1}$ peak ionisation feature can therefore be determined as follows:

$$
\mathrm{FWHM}_{\mathrm{IB}}=\sqrt{\mathrm{FWHM}_{\mathrm{G}}^{2}-\mathrm{FWHM}_{\mathrm{IRF}^{2}}}
$$

where the IRF subscript denotes our experimentally determined energy resolution or instrumental response function (see the Experimental section). Adopting our $\sim 635 \mathrm{eV}$ photon energy FWHM $_{\text {IRF }}$ value of $0.41 \pm 0.01 \mathrm{eV}$ and using eqn (3), we determine a $\mathrm{FWHM}_{\mathrm{IB}}$ value of $0.7 \pm 0.1 \mathrm{eV}$. Feeding this result into eqn (2) along with a $278 \mathrm{~K}$ liquid jet temperature (considering our experimental uncertainties, $k_{\mathrm{B}} T$ at $277 \mathrm{~K}$ and $278 \mathrm{~K}$ differs by a negligible $10^{-4} \mathrm{eV}$ ), we extract an associated $\lambda_{\text {ox }}$ value of $1.8 \pm 0.2 \mathrm{eV}$ or $170 \pm 20 \mathrm{~kJ} \mathrm{~mol}^{-1}$.

Inserting our $\lambda_{\text {ox }}$ estimate and the lowest VIE of $\mathrm{MnO}_{4}{ }^{-}$(aq.) (9.22 $\pm 0.08 \mathrm{eV}$, see Table 1) into expression (1) yields an $\mathrm{MnO}_{4}{ }^{-}$(aq.) $\mathrm{AIE}$ and $\Delta G_{\text {ox }}$ value of $7.4 \pm 0.2 \mathrm{eV}$ or $720 \pm$ $20 \mathrm{~kJ} \mathrm{~mol}^{-1}$. Importantly, we can use this (and other) $\operatorname{AIE}_{\text {(aq.) }}$ result(s) to determine additional thermodynamic quantities of the $\mathrm{MnO}_{4}{ }^{-} / \mathrm{MnO}_{4}{ }^{\bullet}+\mathrm{e}^{-}$system and/or connect the energetics of different chemical species to those of $\mathrm{MnO}_{4}{ }^{-}$(aq.) , examples of which we present below.

The extracted $\operatorname{AIE}_{(\text {aq. }}$ value permits us to estimate the $\operatorname{VEA}_{(\text {aq. }}$ of $\mathrm{MnO}_{4}{ }^{\bullet}$ using the following expression, again within the linear response approximation: ${ }^{14,78}$

$$
\mathrm{VEA}=2 \mathrm{AIE}-\mathrm{VIE}
$$

Using eqn (4) and our direct XPS results, we extract a $\operatorname{VEA}_{(\text {aq. }}$ estimate of $5.7 \pm 0.2 \mathrm{eV}$ or $550 \pm 20 \mathrm{~kJ} \mathrm{~mol}^{-1}$. Furthermore, by comparing our $\mathrm{MnO}_{4}{ }^{-} \mathrm{AIE}_{(\text {aq.) }}$ value with its gas-phase counterpart, we can estimate the $\mathrm{MnO}_{4}{ }^{-}$anion Gibbs free energy of hydration, $\Delta G_{\text {hyd }}$. In Fig. $7 \mathrm{~B}$ we have energetically aligned the AIE value of gas-phase $\mathrm{MnO}_{4}{ }^{-}$to the aqueous-phase value, assuming vacuum level equivalence in liquid- and gas-phase-PES experiments. With such an alignment, Fig. 7B highlights that the difference between $\operatorname{AIE}_{(\text {aq. })}$ and $\operatorname{AIE}_{(\mathrm{g})}$ is equal to $\Delta G_{\text {hyd }}$. Using the gas phase $\mathrm{MnO}_{4}{ }^{-}$AIE of $4.80 \pm 0.1 \mathrm{eV}$, as determined by Gutsev et al. ${ }^{34}$ we estimate a $\Delta G_{\text {hyd }}$ value of $7.4 \pm$ $0.2 \mathrm{eV}-4.8 \pm 0.1 \mathrm{eV}=2.6 \pm 0.2 \mathrm{eV}$, or $260 \pm 20 \mathrm{~kJ} \mathrm{~mol}^{-1}$. With fully solvated $\mathrm{MnO}_{4}{ }^{-}$(aq.) ions expected to form an average of 3.7 hydrogen bonds in solution, ${ }^{79}$ we can further estimate average $\mathrm{MnO}_{4}{ }^{-}$(aq.) hydrogen-bond energies of $0.7 \pm 0.1 \mathrm{eV}$.

Based on the $\mathrm{MnO}_{4}{ }^{-}$(aq.) $1 \mathrm{t}_{1}{ }^{-1}$ ionising transition analysis above and the $4 \mathrm{t}_{2}$ BE and peak width extracted in our RPES analysis, we can similarly estimate the $\lambda_{\text {ox }}$ and AIE values for the first electronically excited state of the $\mathrm{MnO}_{4}{ }^{\bullet}$ (aq.) radical. In 
Table 5 Summary of the valence state energetic and associated thermodynamic parameters extracted from the $\mathrm{MnO}_{4}{ }^{-}$(aq.) liquid-jet PES data. Photooxidised radical state labels are appended to the table, with the dominant molecular orbital configuration changes with respect to the $\mathrm{MnO}_{4}{ }^{-}$(aq.) $\mathrm{ground}^{-}$

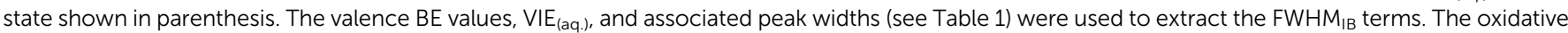
reorganisation energies, $\lambda_{\mathrm{ox}}$, and adiabatic ionisation energies, $\mathrm{AlE}_{(\mathrm{aq}) \text {.) }}$ were extracted using the latter values. The extracted Gibbs free energy of hydration, $\Delta G_{\text {hyd }}$, determined by comparison of the lowest aqueous-phase AlEs to corresponding gas-phase data, is also shown. See the main body of the text for more details

\begin{tabular}{llllr}
\hline Radical state & $\mathrm{VIE}_{(\mathrm{aq} .)} / \mathrm{eV}$ & $\mathrm{FWHM}_{\mathrm{IB}} / \mathrm{eV}$ & $\lambda_{\text {ox }} / \mathrm{eV}$ & $\mathrm{AIE}(\mathrm{aq})$. \\
\hline$(1)^{2} \mathrm{~T}_{1}\left(1 \mathrm{t}_{1}{ }^{-1}\right)$ & $9.22 \pm 0.08$ & $0.7 \pm 0.1$ & $1.8 \pm 0.2$ & $7.4 \pm 0.2$ \\
$(1)^{2} \mathrm{~T}_{2}\left(4 \mathrm{t}_{2}{ }^{-1}\right)$ & $9.85 \pm 0.08$ & $0.6 \pm 0.2$ & $1.5 \pm 0.5$ & $8.4 \pm 0.5$ \\
\hline
\end{tabular}

this case our results correspond to a $283 \mathrm{~K}$ jet temperature (see Table 1) but are still comparable with our 277-278 $\mathrm{K} \mathrm{MnO}_{4}{ }^{\bullet}$ (aq.) ground state results (such a temperature variation leads to a less than $40 \mathrm{meV}$ difference in $\lambda_{\text {ox }}$ and AIE values, well below our reported experimental uncertainties). The results of such an analysis, as well as the aforementioned findings reported in this section, are summarised in Table 5. Here, the energetics are presented along with the electronic state symmetries of the produced $\mathrm{MnO}_{4}{ }^{\bullet}$ (aq.) radicals (assuming a retention of $T_{\mathrm{d}}$ symmetry upon ionisation), with the assigned dominant molecular orbital symmetry of the removed electron given in parenthesis.

As noted in the introduction, a molecular orbital or singleconfiguration electronic structure formalism provides only a qualitative description of the electronic structure of the permanganate anion, with a multi-reference electronic structure treatment demonstrably being needed to quantitatively describe electronically excited states of $\mathrm{MnO}_{4}{ }^{-54}$ Accordingly, we present our valence band spectroscopic results within a more general framework in Fig. 7C, as an electronic state energy level diagram of $\mathrm{MnO}_{4}{ }^{-}$(aq.) and $\mathrm{MnO}_{4}{ }^{\circ}$ (aq.) . The diagram is energetically referenced to the vibronic ground state of $\mathrm{MnO}_{4}{ }^{-}$(aq.) and based on the energetics summarised in Table 5. Otherwise, vertical excitation energies for the electronically excited anion states were determined in our UV-visible absorption spectroscopy measurements (see ESI, $\dagger$ Fig. S1). The VIE values extracted directly from the nonresonant PES/RPES results and the AIE values determined from the associated BEs and peak widths are labelled 'Vertical' and 'Adiabatic' in Fig. 7C, respectively.

The $\operatorname{AIE}_{(\text {aq.) }}$ values (equivalent to $\Delta G_{\text {ox }}$ ) and associated state energetics extracted here are usefully pinned to a universal referencing standard (the vacuum level). They are expected to permit direct comparison between the electronic energetics of $\mathrm{MnO}_{4}{ }^{-}$(aq.) and redox reaction partner species (including other aqueous manganese oxides). Accordingly, complementary liquid-jet-PES experiments will enable (full, rather than just half) reaction free energies to be experimentally determined and chemical process propensities to be predicted. Similarly, the ionisation and state energetics $\left(\mathrm{VIE}_{(\text {aq. })}, \mathrm{AIE}_{(\text {aq. })} / \Delta G_{\text {ox }}, \mathrm{VEA}_{(\text {aq. }}\right)$, and $\Delta G_{\text {hyd }}$ values) reported here are expected to provide a stringent test for emerging multi-configurational electronic structure calculation methods that aspire to explicitly treat and elucidate the effects of hydration on complex solutes. ${ }^{80-83}$ We hope that the results presented in this section in particular will stimulate such theoretical investigations.

\section{Conclusions}

We have presented a comprehensive analysis of the electronic structure of aqueous-phase $\mathrm{MnO}_{4}{ }^{-}$(aq.) and $\mathrm{MnO}_{4}{ }^{\circ}$ (aq.) based on liquid jet direct PES and RPES experiments. Absolute electronic $\mathrm{BE} / \mathrm{VIE}_{(\text {aq.) }}$ values were determined for the majority of the valence manifold, as well as select core levels of $\mathrm{MnO}_{4}{ }^{-}$(aq.) This allowed us to produce an associated experimentallydetermined-BE-scaled, near-complete molecular orbital diagram for this system. At $0.2 \mathrm{M}$ concentrations, similar electronic energetics were identified (relative to the vacuum level) in the aqueous bulk and at the solution-gas interface, as well as upon switching the $\mathrm{MnO}_{4}{ }^{-}$(aq.) counter-ion from $\mathrm{Na}^{+}$(aq.) to $\mathrm{K}^{+}$(aq.) . The aqueous and solid phase BEs of $\mathrm{MnO}_{4}{ }^{-}$were found to be similar, albeit with the aqueous phase BEs shifted to lower values by up to a few-hundred meV compared to their solid-state counterparts. Our aqueous phase results also highlight an increase of the lowest-energy ionising transition BEs by more than $4 \mathrm{eV}$ upon hydration, i.e. in moving from the gas to the aqueous phase.

Our RPES experiments reinforced our direct PES results and allowed us to isolate additional valence BEs that were otherwise obscured by other solute and/or solvent-associated signals. Within a molecular orbital framework, the RPES data additionally revealed specific atomic parentages of the $\mathrm{MnO}_{4}^{-}$(aq.) valence molecular orbitals as well as hybridisation of the constituent outer and inner valence atomic orbitals. Furthermore, $\mathrm{MnO}_{4}{ }^{-}$(aq.) ${ }^{-} \mathrm{H}_{2} \mathrm{O}_{(1)}$ ICD processes were identified, highlighting electronic coupling - and perhaps covalent bonding interactions - between the valence manifolds of the solute and solvent. Finally, careful analysis of our cumulative results allowed us to infer oxidative reorganisation energies, and adiabatic valence $\mathrm{MnO}_{4}{ }^{-}$(aq.) ionisation energies of the two lowest energy ionising transitions. An associated Gibbs free energy of oxidation of $\mathrm{MnO}_{4}{ }^{-}$(aq.) of $7.4 \pm 0.2 \mathrm{eV}\left(720 \pm 20 \mathrm{~kJ} \mathrm{~mol}^{-1}\right)$ was revealed. We additionally extracted a vertical electron affinity estimate of $5.7 \pm 0.2 \mathrm{eV}$ for the $\mathrm{MnO}_{4}{ }^{\circ}$ (aq.) radical. Finally, the Gibbs free energy of hydration of isolated $\mathrm{MnO}_{4}{ }^{-}$was most accurately determined to be $2.6 \pm 0.2 \mathrm{eV}$ or $260 \pm 20 \mathrm{~kJ} \mathrm{~mol}^{-1}$.

Our absolute energy scale measurements are furthermore expected to permit direct comparison between the electronic energetics of $\mathrm{MnO}_{4}{ }^{-}$(aq.), other aqueous manganese oxide species, and/or redox reactive partners (be they bulk aqueousphase reactants or interfacial species/materials). Hence, this work feeds into a larger effort to experimentally characterise not just half-reactions, but complete chemical transformations 
in aqueous solution and on an absolute energy scale. Furthermore, the results presented here constitute experimental benchmarks for state-of-the-art electronic structure calculations and associated spectral simulation methods. Calculations focused on the explicit treatment of hydration and its associated effects on electronically complex solutes ${ }^{80-83}$ as well as those focused on simulating X-ray spectra of increasingly complex systems ${ }^{84-86}$ all stand to benefit. Such studies, although technically challenging, are pre-requisite for the development of a deeper understanding of the energetics, dynamics, and reactivity of this unusual and ubiquitously applied aqueous transition-metal complex.

\section{Conflicts of interest}

There are no conflicts to declare.

\section{Acknowledgements}

The authors gratefully acknowledge the Helmholtz-Zentrum Berlin für Materialien und Energie and the Deutsches Elektronen-Synchrotron for the allocation of synchrotron radiation beamtime, the Chemistry Lab User Support teams at BESSY II and PETRA III, Mr Garlef Wartner for support during the BESSY II measurements, and Mr Sebastian Malerz and Dr Florian Trinter for support during the PETRA III measurements. We also acknowledge Prof. Stephan Thürmer for insightful discussions regarding aqueous-phase PES background correction methodologies. We also gratefully acknowledge Prof. Jógvan Magnus Haugaard Olsen for his support in sharing the optimised $\mathrm{MnO}_{4}^{-}{ }^{-}$(aq.) nuclear geometry files associated with ref. 80. RS thanks the German Research Foundation for granting an Emmy-Noether stipend (SE 2253/3-1).

\section{Notes and references}

1 C. C. Liang, in Encyclopedia of Electrochemistry of the elements, ed. A. J. Bard, Marcel Dekker, Inc., 1973, vol. I.

2 N. Singh and D. G. Lee, Org. Process Res. Dev., 2001, 5, 599-603.

3 M. S. Roderick, J. L. Adcock, J. M. Terry, Z. M. Smith, S. Parry, S. M. Linton, M. T. Thornton, C. J. Barrow and P. S. Francis, J. Phys. Chem. C, 2013, 117, 10286-10293.

4 J. L. Adcock, N. W. Barnett, C. J. Barrow and P. S. Francis, Anal. Chim. Acta, 2014, 807, 9-28.

5 Y. Chang, Y. Bai and J. Qu, Chemosphere, 2016, 163, 73-80.

6 B. Liu, F. Qu, W. Chen, H. Liang, T. Wang, X. Cheng, H. Yu, G. Li and B. Van der Bruggen, Water Res., 2017, 125, 72-80.

7 P. Xie, J. Ma, J. Fang, Y. Guan, S. Yue, X. Li and L. Chen, Environ. Sci. Technol., 2013, 47, 14051-14061.

8 A. J. Gibson, K. G. Latham, R. C. Burns and S. W. Donne, Electrochim. Acta, 2017, 236, 198-211.

9 A. N. Colli, P. Peljo and H. H. Girault, Chem. Commun., 2016, 52, 14039-14042.
10 T. R. Eliato, G. Pazuki and N. Majidian, Energy Sources, Part A, 2016, 38, 644-651.

11 R. A. Marcus, Rev. Mod. Phys., 1993, 65, 599-610.

12 J. Moens, R. Seidel, P. Geerlings, M. Faubel, B. Winter and J. Blumberger, J. Phys. Chem. B, 2010, 114, 9173-9182.

13 R. Seidel, S. Thürmer, J. Moens, P. Geerlings, J. Blumberger and B. Winter, J. Phys. Chem. B, 2011, 115, 11671-11677.

14 D. Ghosh, A. Roy, R. Seidel, B. Winter, S. Bradforth and A. I. Krylov, J. Phys. Chem. B, 2012, 116, 7269-7280.

15 C. Adriaanse, M. Sulpizi, J. VandeVondele and M. Sprik, J. Am. Chem. Soc., 2009, 131, 6046-6047.

16 R. N. Tazhigulov and K. B. Bravaya, J. Phys. Chem. Lett., 2016, 7, 2490-2495.

17 R. Seidel, B. Winter and S. E. Bradforth, Annu. Rev. Phys. Chem., 2016, 67, 283-305.

18 D. Yepes, R. Seidel, B. Winter, J. Blumberger and P. Jaque, J. Phys. Chem. B, 2014, 118, 6850-6863.

19 R. Seidel, K. Atak, S. Thürmer, E. F. Aziz and B. Winter, J. Phys. Chem. B, 2015, 119, 10607-10615.

20 Y. Jiao, B. W. Adams, A. O. Dohn, K. B. Møller, H. Jónsson and C. Rose-Petruck, Phys. Chem. Chem. Phys., 2017, 19, 27266-27274.

21 A. J. Orr-Ewing, Chem. Soc. Rev., 2017, 46, 7597-7614.

22 B. Mennucci, J. M. Martínez and J. Tomasi, J. Phys. Chem. A, 2001, 105, 7287-7296.

23 Y. Marcus, Chem. Rev., 2009, 109, 1346-1370.

24 J. C. Hindman, J. Chem. Phys., 1962, 36, 1000-1016.

25 M. Kubin, M. Guo, T. Kroll, H. Löchel, E. Källman, M. L. Baker, R. Mitzner, S. Gul, J. Kern, A. Föhlisch, A. Erko, U. Bergmann, V. Yachandra, J. Yano, M. Lundberg and P. Wernet, Chem. Sci, 2018, 9, 6813-6829.

26 G. Aullón and S. Alvarez, Theor. Chem. Acc., 2009, 123, 67-73.

27 R. Seidel, S. Thürmer and B. Winter, J. Phys. Chem. Lett., 2011, 2, 633-641.

28 N. Ottosson, M. Faubel, S. E. Bradforth, P. Jungwirth and B. Winter, J. Electron Spectrosc. Relat. Phenom., 2010, 177, 60-70.

29 Y.-I. Suzuki, K. Nishizawa, N. Kurahashi and T. Suzuki, Phys. Rev. E: Stat., Nonlinear, Soft Matter Phys., 2014, 90, 010302.

30 B. Winter, Nucl. Instrum. Methods Phys. Res., Sect. A, 2009, 601, 139-150.

31 T. Miteva, N. V. Kryzhevoi, N. Sisourat, C. Nicolas, W. Pokapanich, T. Saisopa, P. Songsiriritthigul, Y. Rattanachai, A. Dreuw, J. Wenzel, J. Palaudoux, G. Öhrwall, R. Püttner, L. S. Cederbaum, J.-P. Rueff and D. Céolin, J. Phys. Chem. Lett., 2018, 9, 4457-4462.

32 H. Ali, R. Seidel, M. N. Pohl and B. Winter, Chem. Sci., 2018, 9, 4511-4523.

33 E. F. Aziz, N. Ottosson, M. Faubel, I. V. Hertel and B. Winter, Nature, 2008, 455, 89-91.

34 G. Gutsev, B. K. Rao, P. Jena, X.-B. Wang and L.-S. Wang, Chem. Phys. Lett., 1998, 312, 598-605.

35 M. Oku, J. Electron Spectrosc. Relat. Phenom., 1995, 74, 135-148. 36 M. Oku, T. Shishido and S. Kohiki, Surf. Sci. Spectra, 2004, 11, 59-65. 
37 F. Reinert, S. Kumar, P. Steiner, R. Claessen and S. Hüfner, Z. Phys. B: Condens. Matter, 1994, 94, 431-438.

38 S. G. Minasian, J. M. Keith, E. R. Batista, K. S. Boland, J. A. Bradley, S. R. Daly, S. A. Kozimor, W. W. Lukens, R. L. Martin, D. Nordlund, G. T. Seidler, D. K. Shuh, D. Sokaras, T. Tyliszczak, G. L. Wagner, T.-C. Weng and P. Yang, J. Am. Chem. Soc., 2013, 135, 1864-1871.

39 T. Ziegler, A Chronicle About the Development of Electronic Structure Theories for Transition Metal Complexes, 2011.

40 L. Jose, M. Seth and T. Ziegler, J. Phys. Chem. A, 2012, 116, 1864-1876.

41 K. H. Johnson and F. C. Smith, Phys. Rev. B: Solid State, 1972, 5, 831-843.

42 Helmholtz-Zentrum Berlin für Materialien und Energie, J. Large-scale Res. Facil., 2016, 2, A72.

43 R. Seidel, M. N. Pohl, H. Ali, B. Winter and E. F. Aziz, Rev. Sci. Instrum., 2017, 88, 073107.

44 J. Viefhaus, F. Scholz, S. Deinert, L. Glaser, M. Ilchen, J. Seltmann, P. Walter and F. Siewert, Nucl. Instrum. Methods Phys. Res., Sect. A, 2013, 710, 151-154.

45 http:/www.fhi-berlin.mpg.de/mp/winter/Main/Experimental Methods, accessed 02.07, 2020.

46 G. Zimmerman, J. Chem. Phys., 1955, 23, 825-832.

47 S. K. Eriksson, M. Hahlin, J. M. Kahk, I. J. Villar-Garcia, M. J. Webb, H. Grennberg, R. Yakimova, H. Rensmo, K. Edström, A. Hagfeldt, H. Siegbahn, M. O. M. Edwards, P. G. Karlsson, K. Backlund, J. Åhlund and D. J. Payne, Rev. Sci. Instrum., 2014, 85, 075119.

48 S. Thürmer, R. Seidel, M. Faubel, W. Eberhardt, J. C. Hemminger, S. E. Bradforth and B. Winter, Phys. Rev. Lett., 2013, 111, 173005.

49 N. Kurahashi, S. Karashima, Y. Tang, T. Horio, B. Abulimiti, Y.-I. Suzuki, Y. Ogi, M. Oura and T. Suzuki, J. Chem. Phys., 2014, 140, 174506.

50 S. Hellmann, C. Sohrt, M. Beye, T. Rohwer, F. Sorgenfrei, M. Marczynski-Bühlow, M. Kalläne, H. Redlin, F. Hennies, M. Bauer, A. Föhlisch, L. Kipp, W. Wurth and K. Rossnagel, New J. Phys., 2012, 14, 013062.

51 D. A. Shirley, Phys. Rev. B: Solid State, 1972, 5, 4709-4714.

52 B. Winter, E. F. Aziz, U. Hergenhahn, M. Faubel and I. V. Hertel, J. Chem. Phys., 2007, 126, 124504.

53 L. Å. Näslund, J. Lüning, Y. Ufuktepe, H. Ogasawara, P. Wernet, U. Bergmann, L. G. M. Pettersson and A. Nilsson, J. Phys. Chem. B, 2005, 109, 13835-13839.

54 N. M. S. Almeida, R. G. McKinlay and M. J. Paterson, Chem. Phys., 2015, 446, 86-91.

55 T. Ziegler, in Molecular Electronic Structures of Transition Metal Complexes II, ed. D. M. P. Mingos, P. Day and J. P. Dahl, Springer Berlin Heidelberg, Berlin, Heidelberg, 2012, pp. 1-38, DOI: 10.1007/430_2011_47.

56 P. Sharma, D. G. Truhlar and L. Gagliardi, J. Chem. Phys., 2018, 148, 124305.

57 T. K. Sham and B. S. Brunschwig, J. Am. Chem. Soc., 1981, 103, 1590-1591.

58 W. Kiefer and H. J. Bernstein, Mol. Phys., 1972, 23, 835-851.
59 M. N. Pohl, E. Muchová, R. Seidel, H. Ali, Š. Sršeň, I. Wilkinson, B. Winter and P. Slavíček, Chem. Sci., 2019, 10, 848-865.

60 B. Winter, R. Weber, W. Widdra, M. Dittmar, M. Faubel and I. V. Hertel, J. Phys. Chem. A, 2004, 108, 2625-2632.

61 B. Winter, R. Weber, I. V. Hertel, M. Faubel, P. Jungwirth, E. C. Brown and S. E. Bradforth, J. Am. Chem. Soc., 2005, 127, 7203-7214.

62 A. P. Mortola, H. Basch and J. W. Moskowitz, Int. J. Quantum Chem., 1973, 7, 725-737.

63 M. C. Biesinger, B. P. Payne, A. P. Grosvenor, L. W. M. Lau, A. R. Gerson and R. S. C. Smart, Appl. Surf. Sci., 2011, 257, 2717-2730.

64 J. K. A. C. Thompson, D. T. Atwood, E. M. Gullikson, M. R. Howells, J. B. Kortright, Y. Liu, A. L. Robinson, J. H. Underwood, K. Kim, I. Lindau, P. Pianetta, H. Winick, G. P. Williams and J. H. Scofield, X-Ray Data Booklet, Lawrence Berkeley National Laboratory, University of California, Berkeley, California 94720, 3rd edn, 2009.

65 W. Haynes, CRC Handbook of Chemistry and Physics, CRC Press, 92nd edn, 2011.

66 A. Pflueger, Ann. Phys., 1903, 12, 430.

67 F. Reinert, P. Steiner, R. Zimmermann, R. Claessen and S. Hüfner, Z. Phys. B: Condens. Matter, 1995, 99, 229-234.

68 M. A. Brown, M. Faubel and B. Winter, Annu. Rep. Prog. Chem., Sect. C: Phys. Chem., 2009, 105, 174-212.

69 J. L. Campbell and T. Papp, At. Data Nucl. Data Tables, 2001, 77, 1-56.

70 I. Unger, R. Seidel, S. Thürmer, M. N. Pohl, E. F. Aziz, L. S. Cederbaum, E. Muchová, P. Slavíček, B. Winter and N. V. Kryzhevoi, Nat. Chem., 2017, 9, 708-714.

71 Z.-H. Loh, G. Doumy, C. Arnold, L. Kjellsson, S. H. Southworth, A. Al Haddad, Y. Kumagai, M.-F. Tu, P. J. Ho, A. M. March, R. D. Schaller, M. S. Bin Mohd Yusof, T. Debnath, M. Simon, R. Welsch, L. Inhester, K. Khalili, K. Nanda, A. I. Krylov, S. Moeller, G. Coslovich, J. Koralek, M. P. Minitti, W. F. Schlotter, J.-E. Rubensson, R. Santra and L. Young, Science, 2020, 367, 179-182.

72 S. Thürmer, I. Unger, P. Slavíček and B. Winter, J. Phys. Chem. C, 2013, 117, 22268-22275.

73 B. Winter, U. Hergenhahn, M. Faubel, O. Björneholm and I. Hertel, J. Chem. Phys., 2007, 127, 094501.

74 S. Thürmer, R. Seidel, W. Eberhardt, S. E. Bradforth and B. Winter, J. Am. Chem. Soc., 2011, 133, 12528-12535.

75 M. Oku, J. Electron Spectrosc. Relat. Phenom., 1995, 74, 135-148. 76 D. Coster and R. D. L. Kronig, Physica, 1935, 2, 13-24.

77 P. Winget, E. J. Weber, C. J. Cramer and D. G. Truhlar, Phys. Chem. Chem. Phys., 2000, 2, 1231-1239.

78 Y. Tateyama, J. Blumberger, M. Sprik and I. Tavernelli, J. Chem. Phys., 2005, 122, 234505.

79 M. Bühl, J. Phys. Chem. A, 2002, 106, 10505-10509.

80 J. M. Olsen and E. Hedegård, Phys. Chem. Chem. Phys., 2017, 19, 15870-15875.

81 C. Bistafa, Y. Kitamura, M. Nagaoka and S. Canuto, J. Phys. Chem. B, 2018, 122, 9202-9209.

82 D. Asthagiri, L. R. Pratt, M. E. Paulaitis and S. B. Rempe, J. Am. Chem. Soc., 2004, 126, 1285-1289. 
83 M. Uudsemaa and T. Tamm, Chem. Phys. Lett., 2004, 400, 54-58. 84 S. Sreekantan Nair Lalithambika, R. Golnak, B. Winter and K. Atak, Inorg. Chem., 2019, 58, 4731-4740.

85 S. S. N. Lalithambika, K. Atak, R. Seidel, A. Neubauer, T. Brandenburg, J. Xiao, B. Winter and E. F. Aziz, Sci. Rep., 2017, 7, 40811.
86 E. Pluhařová, C. Schroeder, R. Seidel, S. E. Bradforth, B. Winter, M. Faubel, P. Slavíček and P. Jungwirth, J. Phys. Chem. Lett., 2013, 4, 3766-3769.

87 J. M. H. Olsen and E. D. Hedegård, Phys. Chem. Chem. Phys., 2017, 19, 15870-15875.

88 W. F. Egelhoff, Surf. Sci. Rep., 1987, 6, 253-415. 